\title{
18F PET with flutemetamol for the early diagnosis of Alzheimer's disease dementia and other dementias in people with mild cognitive impairment (MCI) (Review)
}

Martínez G, Vernooij RWM, Fuentes Padilla P, Zamora J, Flicker L, Bonfill Cosp X

Martínez G, Vernooij RWM, Fuentes Padilla P, Zamora J, Flicker L, Bonfill Cosp X.

18 F PET with flutemetamol for the early diagnosis of Alzheimer's disease dementia and other dementias in people with mild cognitive impairment $(\mathrm{MCl})$.

Cochrane Database of Systematic Reviews 2017, Issue 11. Art. No.: CD012884.

DOI: 10.1002/14651858.CD012884.

www.cochranelibrary.com

18F PET with flutemetamol for the early diagnosis of Alzheimer's disease dementia and other dementias in people with mild cognitive impairment (MCI) (Review) 
HEADER 1

ABSTRACT

PLAIN LANGUAGE SUMMARY

SUMMARY OF FINDINGS

BACKGROUND

OBJECTIVES

METHODS

RESULTS

Figure 1.

Figure 2.

Figure 3.

DISCUSSION

AUTHORS' CONCLUSIONS

ACKNOWLEDGEMENTS

REFERENCES

CHARACTERISTICS OF STUDIES

DATA

Test 1. 18F-flutemetamol.

APPENDICES

CONTRIBUTIONS OF AUTHORS

DECLARATIONS OF INTEREST

SOURCES OF SUPPORT

INDEX TERMS

\section{TABLE OF CONTENTS}




\title{
18F PET with flutemetamol for the early diagnosis of Alzheimer's disease dementia and other dementias in people with mild cognitive impairment (MCI)
}

\author{
Gabriel Martínez1,2,3, Robin WM Vernooij ${ }^{1}$, Paulina Fuentes Padilla1,2, Javier Zamora ${ }^{4}$, Leon Flicker5, Xavier Bonfill Cosp 6,7
}

${ }^{1}$ lberoamerican Cochrane Centre, Barcelona, Spain. ${ }^{2}$ Faculty of Medicine and Dentistry, Universidad de Antofagasta, Antofagasta, Chile. ${ }^{3}$ Alzheimer Research Center and Memory Clinic of Fundació ACE, Institut Català de Neurociències Aplicades, Barcelona, Spain. ${ }^{4}$ Clinical Biostatistics Unit, Ramon y Cajal Institute for Health Research (IRYCIS), CIBER Epidemiology and Public Health (CIBERESP), Madrid (Spain) and Women's Health Research Unit, Centre for Primary Care and Public Health, Queen Mary University of London, London, UK. 5 Western Australian Centre for Health \& Ageing - WACHA, University of Western Australia, Perth, Australia. 6 Iberoamerican Cochrane Centre, Biomedical Research Institute Sant Pau (IIB Sant Pau), CIBER Epidemiología y Salud Pública (CIBERESP), Barcelona, Spain. 7Universitat Autònoma de Barcelona, Barcelona, Spain

Contact address: Gabriel Martínez, Iberoamerican Cochrane Centre, C/ Sant Antoni Maria Claret 167, Pavelló 18 Planta 0, Barcelona, Barcelona, 08025, Spain.gmartinez@cochrane.es, gmartinezfuentes@gmail.com.

Editorial group: Cochrane Dementia and Cognitive Improvement Group.

Publication status and date: New, published in Issue 11, 2017.

Citation: Martínez G, Vernooij RWM, Fuentes Padilla P, Zamora J, Flicker L, Bonfill Cosp X. 18F PET with flutemetamol for the early diagnosis of Alzheimer's disease dementia and other dementias in people with mild cognitive impairment (MCI). Cochrane Database of Systematic Reviews 2017, Issue 11. Art. No.: CD012884. DOI: 10.1002/14651858.CD012884.

Copyright @ 2017 The Cochrane Collaboration. Published by John Wiley \& Sons, Ltd.

\section{A B S T R A C T}

\section{Background}

$18 \mathrm{~F}$-flutemetamol uptake by brain tissue, measured by positron emission tomography (PET), is accepted by regulatory agencies like the Food and Drug Administration (FDA) and the European Medicine Agencies (EMA) for assessing amyloid load in people with dementia. Its added value is mainly demonstrated by excluding Alzheimer's pathology in an established dementia diagnosis. However, the National Institute on Aging and Alzheimer's Association (NIA-AA) revised the diagnostic criteria for Alzheimer's disease and the confidence in the diagnosis of mild cognitive impairment $(\mathrm{MCl})$ due to Alzheimer's disease may be increased when using some amyloid biomarkers tests like $18 \mathrm{~F}$-flutemetamol. These tests, added to the $\mathrm{MCl}$ core clinical criteria, might increase the diagnostic test accuracy (DTA) of a testing strategy. However, the DTA of $18 \mathrm{~F}$-flutemetamol to predict the progression from MCI to Alzheimer's disease dementia (ADD) or other dementias has not yet been systematically evaluated.

\section{Objectives}

To determine the DTA of the $18 \mathrm{~F}$-flutemetamol PET scan for detecting people with $\mathrm{MCl}$ at time of performing the test who will clinically progress to ADD, other forms of dementia (non-ADD) or any form of dementia at follow-up.

\section{Search methods}

The most recent search for this review was performed in May 2017. We searched MEDLINE (OvidSP), Embase (OvidSP), PsycINFO (OvidSP), BIOSIS Citation Index (Thomson Reuters Web of Science), Web of Science Core Collection, including the Science Citation Index (Thomson Reuters Web of Science) and the Conference Proceedings Citation Index (Thomson Reuters Web of Science), LILACS (BIREME), CINAHL (EBSCOhost), ClinicalTrials.gov (https://clinicaltrials.gov), and the World Health Organization International Clinical Trials Registry Platform (WHO ICTRP) (http://www.who.int/ictrp/search/en/). We also searched ALOIS, the Cochrane Dementia \& Cognitive Improvement Group's specialised register of dementia studies (http://www.medicine.ox.ac.uk/alois/). We checked the reference lists of any relevant studies

18F PET with flutemetamol for the early diagnosis of Alzheimer's disease dementia and other dementias in people with mild cognitive 
and systematic reviews, and performed citation tracking using the Science Citation Index to identify any additional relevant studies. No language or date restrictions were applied to the electronic searches.

\section{Selection criteria}

We included studies that had prospectively defined cohorts with any accepted definition of $\mathrm{MCl}$ at time of performing the test and the use of $18 \mathrm{~F}$-flutemetamol scan to evaluate the DTA of the progression from $\mathrm{MCl}$ to ADD or other forms of dementia. In addition, we only selected studies that applied a reference standard for Alzheimer's dementia diagnosis, for example, National Institute of Neurological and Communicative Disorders and Stroke and the Alzheimer's Disease and Related Disorders Association (NINCDS-ADRDA) or Diagnostic and Statistical Manual of Mental Disorders-IV (DSM-IV) criteria.

\section{Data collection and analysis}

We screened all titles and abstracts identified in electronic-database searches. Two review authors independently selected studies for inclusion and extracted data to create two-by-two tables, showing the binary test results cross-classified with the binary reference standard. We used these data to calculate sensitivities, specificities, and their $95 \%$ confidence intervals. Two independent assessors performed quality assessment using the QUADAS-2 tool plus some additional items to assess the methodological quality of the included studies.

\section{Main results}

Progression from $\mathrm{MCl}$ to ADD was evaluated in 243 participants from two studies. The studies reported data on 19 participants with two years of follow-up and on 224 participants with three years of follow-up. Nine (47.4\%) participants converted at two years follow-up and $81(36.2 \%)$ converted at three years of follow-up.

There were concerns about participant selection and sampling in both studies. The index test domain in one study was considered unclear and in the second study it was considered at low risk of bias. For the reference standard domain, one study was considered at low risk and the second study was considered to have an unclear risk of bias. Regarding the domains of flow and timing, both studies were considered at high risk of bias.

$\underline{\mathrm{MCI}}$ to ADD;

Progression from $\mathrm{MCl}$ to $\mathrm{ADD}$ at two years of follow-up had a sensitivity of $89 \%$ ( $95 \% \mathrm{Cl} 52$ to 100 ) and a specificity of $80 \%$ (95\% $\mathrm{Cl} 44$ to 97) by quantitative assessment by SUVR ( $n=19,1$ study).

Progression from $\mathrm{MCl}$ to $\mathrm{ADD}$ at three years of follow-up had a sensitivity of $64 \%$ (95\% $\mathrm{Cl} 53$ to 75 ) and a specificity of $69 \%$ (95\% $\mathrm{Cl} 60$ to 76) by visual assessment ( $n=224,1$ study).

There was no information regarding the other two objectives in this systematic review (SR): progression from $\mathrm{MCl}$ to other forms of dementia and progression to any form of dementia at follow-up.

\section{Authors' conclusions}

Due to the varying sensitivity and specificity for predicting the progression from $\mathrm{MCl}$ to ADD and the limited data available, we cannot recommend routine use of $18 \mathrm{~F}$-flutemetamol in clinical practice. $18 \mathrm{~F}$-flutemetamol has high financial costs; therefore, clearly demonstrating its DTA and standardising the process of the $18 \mathrm{~F}$-flutemetamol modality is important prior to its wider use.

\section{PLAIN LANGUAGE SUMMARY}

\section{F PET with flutemetamol for the early diagnosis of Alzheimer's disease dementia and other dementias in people with mild cognitive impairment}

Review question: In people with mild cognitive impairment $(\mathrm{MCl})$, does using a $18 \mathrm{~F}$ PET scan with flutemetamol predict the progression to Alzheimer's disease dementia (ADD) and other dementias?

\section{Background}

Due to global ageing, the number of people with dementia is expected to increase dramatically in the next few decades. Diagnosing dementia at an early stage is desirable, but there is no widespread agreement on the best approach. A range of simple pen and paper tests used by healthcare professionals can assess people with poor memory or cognitive impairment. Whether or not using special PET scans that detect amyloid -one of the hallmarks of Alzheimer's disease- improves our ability to predict the progression from MCI to ADD or other forms of dementia remains unclear. Since these tests are expensive, it is important that they provide additional benefits.

\section{Aim}

We aimed to evaluate the accuracy of the $18 \mathrm{~F}$-flutemetamol PET scan in identifying those people with $\mathrm{MCl}$ who clinically progress to ADD, other types of dementia, or any form of dementia over a period of time.

18F PET with flutemetamol for the early diagnosis of Alzheimer's disease dementia and other dementias in people with mild cognitive 


\section{Study characteristics}

The evidence is current to May 2017. We found two studies evaluating the progression from $\mathrm{MCl}$ to ADD. The studies included $252 \mathrm{MCl}$ eligible participants, with 243 participants that had follow-up. Of these, 127 were women. The average age in one study with two years of follow-up was $72.7 \pm 7.09$ years. In the other study with three years of follow-up, the average age was $71.1 \pm 8.62$ years. The setting in one study was memory clinics.

Study funding sources: both studies were funded by the test manufacturer.

\section{Quality of the evidence}

The main limitation of this review was that our findings were based on only two studies, with not enough details on how the people were selected, how the interpretation of the PET scan was made in one study, how the clinical diagnosis of dementia was established in the other study. The studies were considered to be at high risk of bias due to potential conflicts of interest detected.

\section{Key findings}

In this review, we found that the $18 \mathrm{~F}$-flutemetamol PET scan, as a single test, in one study with 19 participants included with 2 years of follow-up, had a sensitivity of $89 \%$ and a specificity of $80 \%$. This means that in a cohort with 100 participants with $\mathrm{MCl}$ and a proportion of progression in this study of $47 \%$, we would expect 42 of $47 \mathrm{MCl}$ participants with a positive result for $18 \mathrm{~F}$-flutemetamol scan to progress to ADD, and 5 participants to be falsely positive. In addition, we would expect 42 of 53 participants who will not progress to ADD to have a negative result for $18 \mathrm{~F}$-flutemetamol, and 11 to be falsely negative.

In the other study with 224 participants included in the analysis with 3 years follow-up, the sensitivity was $64 \%$ and the specificity was $69 \%$. This means that in a cohort with 100 participants with $\mathrm{MCl}$ and a proportion of progression in this study of $36 \%$, we would expect 23 of 36 $\mathrm{MCl}$ participants with a positive result for ${ }^{18} \mathrm{~F}$-flutemetamol to progress to ADD, and 13 participants to be falsely positive. In addition, we would expect 44 of 64 participants who will not progress to ADD to have a negative result for $18 \mathrm{~F}$-flutemetamol, and 20 to be falsely negative.

There was no information regarding the progression from $\mathrm{MCl}$ to other forms of dementia and progression to any form of dementia at follow-up.

We conclude that ${ }^{18} \mathrm{~F}$-flutemetamol PET imaging cannot be recommended for routine use in clinical practice to predict the progression from $\mathrm{MCl}$ to $\mathrm{ADD}$ based on the currently available data. More studies are needed to clearly demonstrate its usefulness. 


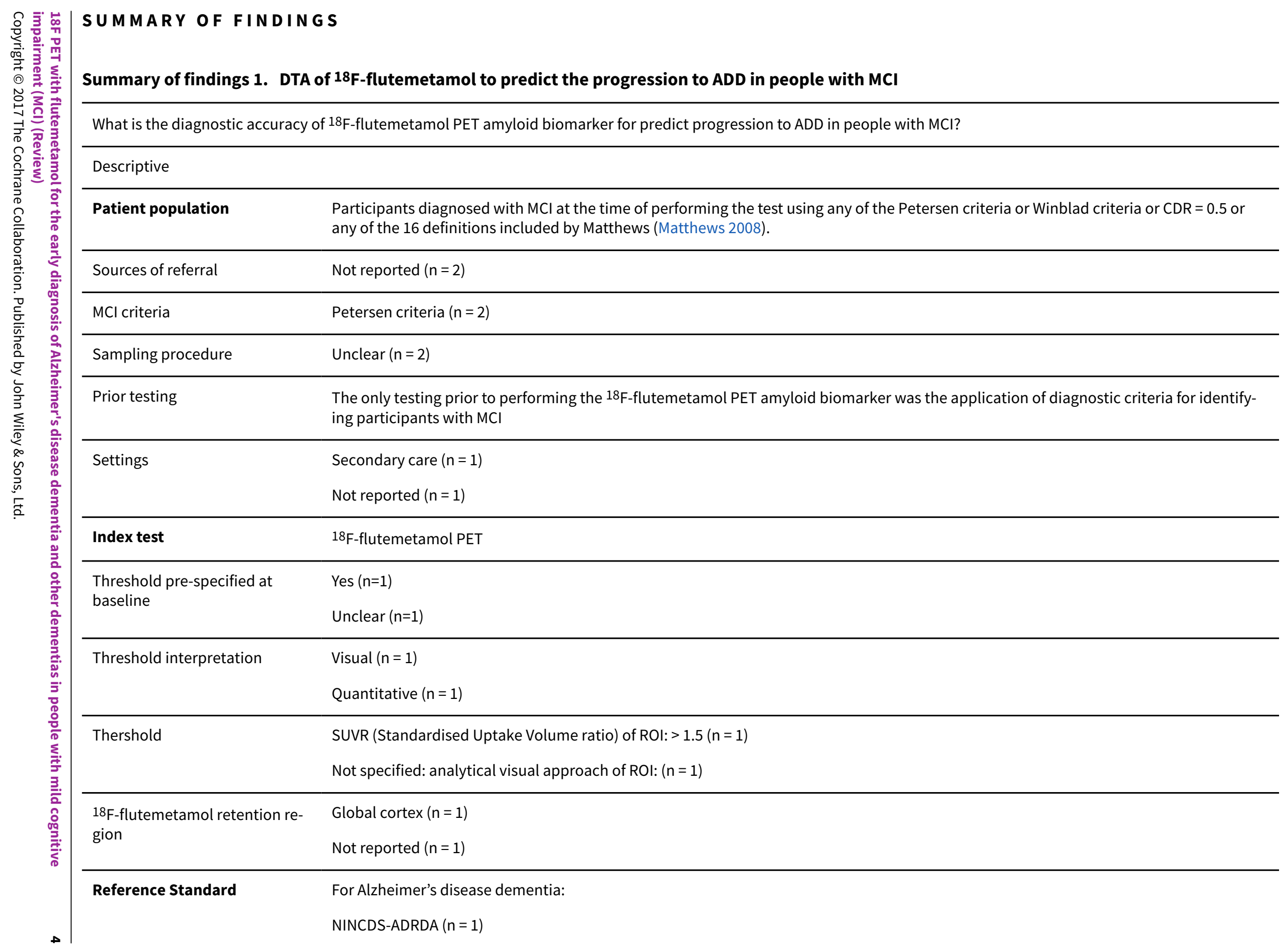




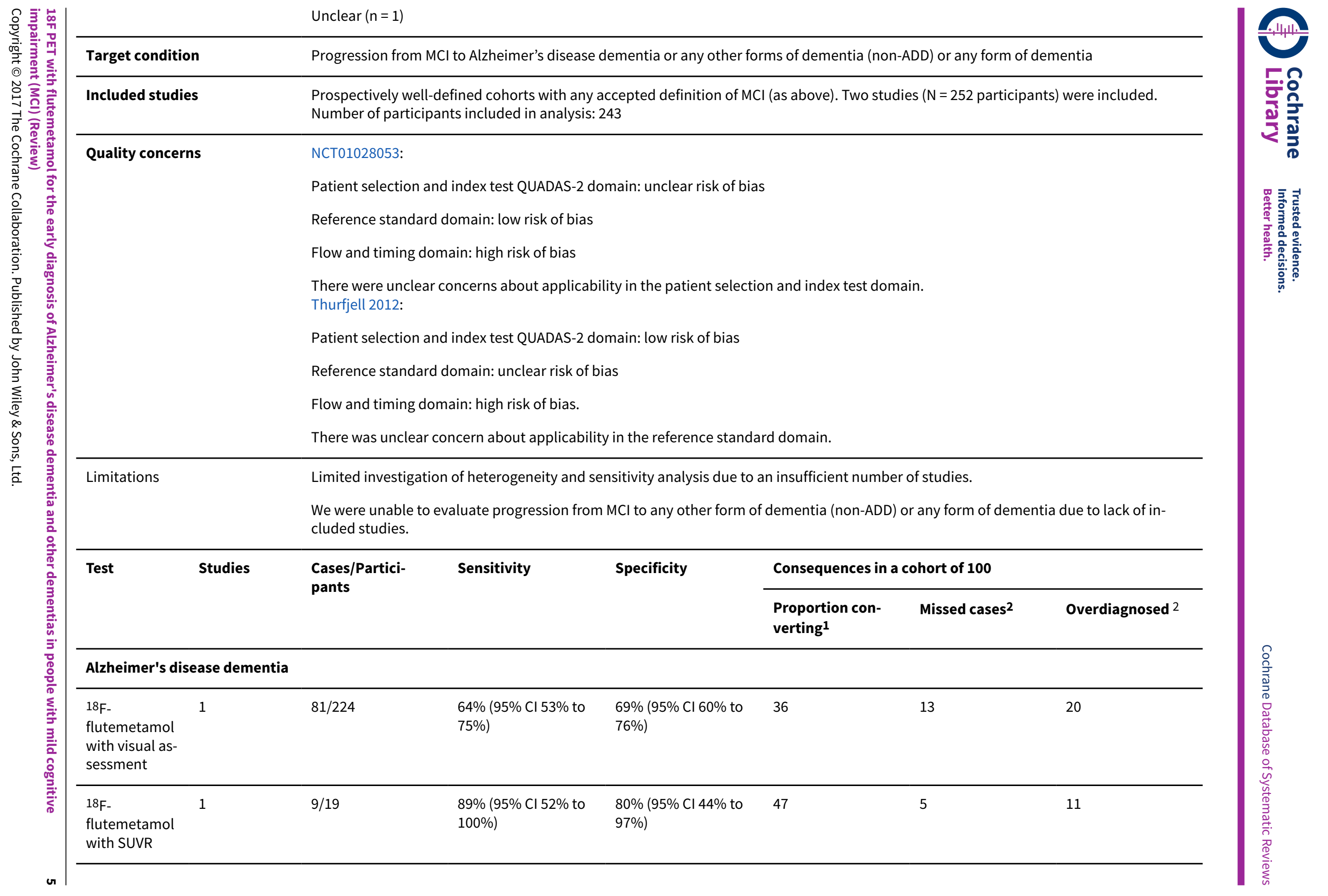




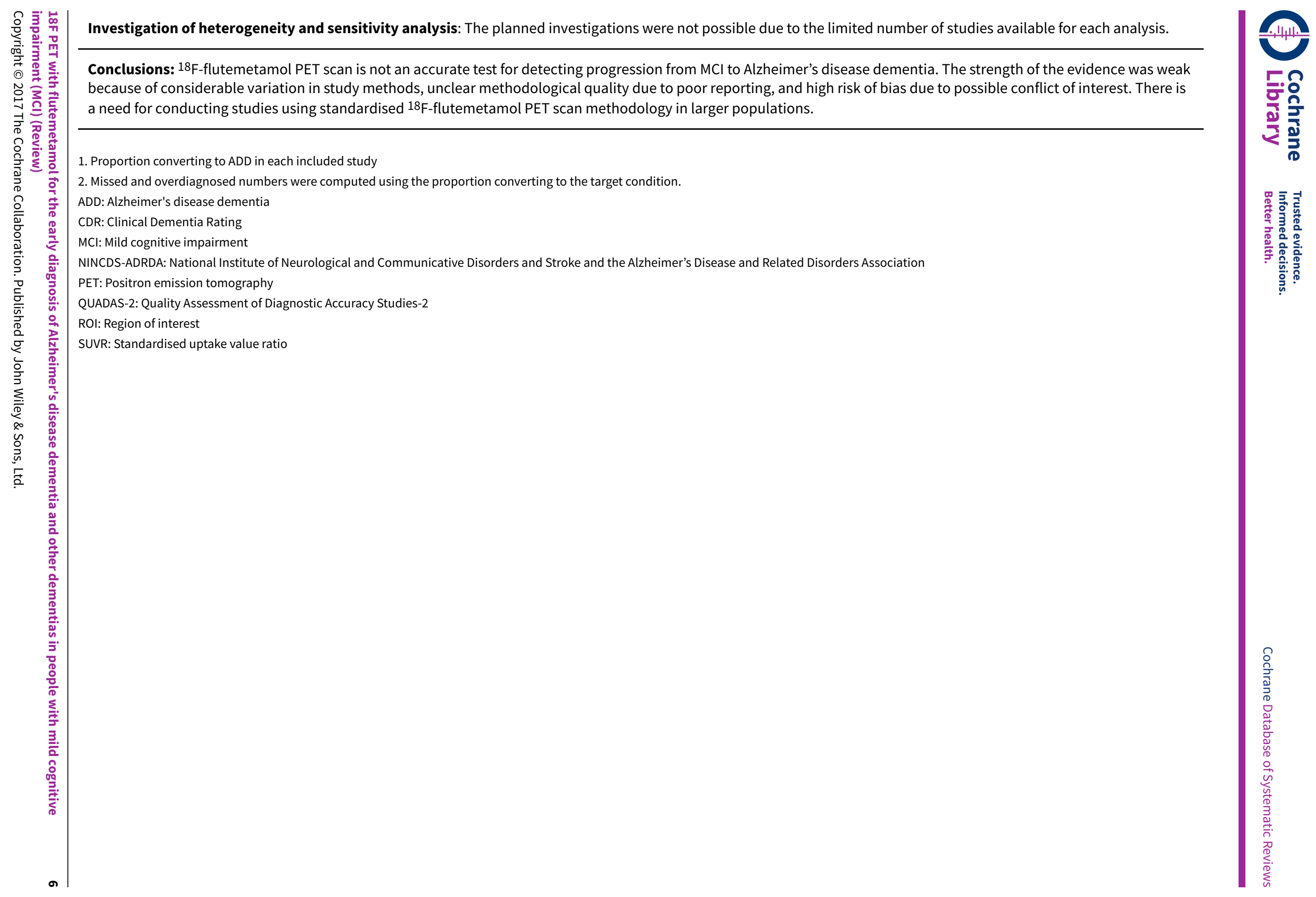




\section{B A C K G R O U N D}

Dementia is a syndrome due to a brain disease - usually of a chronic or progressive nature - in which there is disturbance of multiple higher cortical functions, including memory, thinking, orientation, comprehension, calculation, learning capacity, language, and judgement. However, consciousness remains unaffected. See the glossary in Appendix 1. The impairments of cognitive function are commonly accompanied, and occasionally preceded, by a deterioration in emotional control, social behaviour, motivation, and the impairment is sufficient to interfere with everyday activities. Dementia is a collection of different subtypes distinguished by the underlying pathology. ADD is the most common form of dementia and other important pathologies associated with dementia are vascular disease, Lewy bodies, and frontotemporal pathology (WHO 2012).

Dementia is a serious worldwide public health problem, with a prevalence of $4.7 \%$ in adults older than 60 years $(6.2 \%$ and $6.5 \%$ in Europe and the Americas, respectively). Due to its prevalence in older people, it is expected that the number of people with dementia will increase dramatically. Consequently, in the year 2050, an expected number of 115 million people will have dementia. This will result in a considerable economic burden, which currently stands at $1 \%$ of the world's Gross National Product (GNP) in direct and indirect costs (WHO 2012). These financial costs are in addition to the devastating personal and social consequences of the condition.

The definition of $\mathrm{MCl}$ applies to people without evidence of significant deterioration in activities of daily living, but with subjective memory complaints and cognitive impairment detected by standardised tests. $\mathrm{MCl}$ often precedes clinical dementia, but there is no consensus regarding how to operationalise the $\mathrm{MCl}$ diagnosis. There are several clinical criteria to define which people have $\mathrm{MCl}$, including the Petersen criteria or Petersen Revisited Criteria (Petersen 1999; Petersen 2004; Winblad 2004), Clinical Dementia Rating Scale (CDR= 0.5) (Morris 1993), or 16 other different classifications of $\mathrm{MCl}$ (Matthews 2008).

A diagnosis of $\mathrm{MCl}$ reputedly allows testing of preventive interventions that would slow the progression of $\mathrm{MCl}$ to dementia. If the progression of $\mathrm{MCl}$ to dementia could be deferred by five years, the prevalence of dementia would decrease by $43 \%$ in 2050 (Alzheimer's Association 2010). $\mathrm{MCl}$ has an annual progression rate to ADD from $5 \%$ to $15 \%$. However, not every person with $\mathrm{MCl}$ develops dementia, and a significant number of people recover or stabilise. Therefore, future research should try to clarify which people with $\mathrm{MCl}$ develop dementia in order to be able to focus specifically on people who are at high risk of developing dementia. This may possibly explain the failure of therapy to alter the progression to dementia in people with $\mathrm{MCl}$. Other aspects that may contribute to this failure are the disparity in diagnostic criteria and different settings of the studied participants: community, primary, secondary, and research centres (Bruscoli 2004; Mattsson 2009; Petersen 1999; Petersen 2009).

The definition of Alzheimer's disease pathology is over 100 years old. This pathology includes neuritic plaques that contain deposits of amyloid beta $(A \beta)$ and neurofibrillary tangles (Goedert 2006). This pathology is present in approximately $84 \%$ of all people with dementia (Schneider 2007). Furthermore, Alzheimer's disease pathology is found in $88 \%$ of people diagnosed with probable
ADD (Schneider 2009). Despite this, Alzheimer's disease pathology may be found concomitantly at autopsy in people thought to have other forms of dementia, such as vascular dementia, Lewy body dementia, or frontotemporal dementia (FTD) (Jellinger 2006). Furthermore, at least five common pathologies have been found in the brains of people who died and were thought to have ADD prior to death (White 2009). Also, Alzheimer's disease pathology was found in $42 \%$ of community-dwelling older people without dementia (Schneider 2007). This has generated controversy about the importance of the presence of Alzheimer's disease pathology. The pathology can be associated with aging per se, and, for older people, the relationship between amyloid plaque burden and cognitive impairment diminishes as age progresses (Savva 2009). Thus, this pathology could be an epiphenomenon associated with the presence of dementia, e.g. a by-product of repair mechanisms by vascular damage (De la Torre 2004; Garcia-Alloza 2011). On the other hand, this controversy could be because our clinical diagnostic criteria have not had enough accuracy to diagnose Alzheimer's disease that is detected by histopathology in postmortem studies (Hyman 2012). In addition, other researchers think that there is not a real controversy about the amyloid hypothesis, because the amyloid cascade and the $A \beta$ deposition have a primary role in Alzheimer's disease (Selkoe 2016).

More recently, the development of $A \beta$ pathology biomarkers in vivo has been suggested as an important advance as a diagnostic tool in the field of Alzheimer's disease, and has promoted the creation of new diagnostic criteria for people without symptoms (preclinical stages), people with $\mathrm{MCl}$, and people with $\mathrm{ADD}$, based on the presence of biomarkers of Alzheimer's disease. These have included $A \beta$ tracers by positron emission tomography (PET) (Albert 2011; Dubois 2014; McKhann 2011; Sperling 2011). However, uncertainties regarding the usability of biomarkers in the diagnosis of dementia still exist, mainly due to variation between biomarker types, criteria for positivity, and differences in methodology (NoelStorr 2013). This prompted an important initiative, the Standards for Reporting of Diagnostic Accuracy Studies in dementia studies (STARDdem) statement (Noel-Storr 2014). Consequently, clinical properties of dementia biomarkers should not be assumed, and formal systematic evaluations of sensitivity, specificity, and other properties of biomarkers should be performed (Davis 2013).

PET is an imaging technique using compounds labelled with short-lived positron-emitting radionuclides. The use of $A \beta$ ligands permits the in vivo detection of amyloid deposition in the brain. ${ }^{18} \mathrm{~F}$ PET ligands, such as $18 \mathrm{~F}$-flutemetamol, is a fluorinated tracer, derived from the Pittsburgh Compound $B$ (the first tracer developed), and it is characterised by a higher median life of 110 minutes than the Pittsburgh Compound $B$ and a high affinity for amyloid $\beta$. The performance of the $18 \mathrm{~F}$-flutemetamol PET scan was probed in vivo with healthy people and ADD (Nelissen 2009) and also in people with $\mathrm{MCl}$ (Vandenberghe 2010) and it has been considered that it could eventually be used to differentiate between different dementia types, specifically between FTD and ADD like other fluorinated tracers such as $18 \mathrm{~F}$-florbetaben (Villemagne 2011) or 18 F-florbetapir (Kobylecki 2015).

In 2013, 18F-flutemetamol was approved by the Food and Drug Administration (FDA) and, in 2014, by the European Medicines Agency (EMA). A positive scan indicates moderate to frequent presence of neuritic amyloid plaques. However, this might also occur in people with other neurological conditions and in

18F PET with flutemetamol for the early diagnosis of Alzheimer's disease dementia and other dementias in people with mild cognitive impairment ( $\mathrm{MCI})$ (Review)

Copyright (C) 2017 The Cochrane Collaboration. Published by John Wiley \& Sons, Ltd. 
older adults with normal cognition. Therefore, the safety and effectiveness of $18 \mathrm{~F}$-flutemetamol have not been established for predicting development of dementia or other neurological conditions and it should be combined with other diagnostic evaluations or instruments (EMA 2014; FDA 2014).

Despite not being approved for this purpose by the regulatory agencies, research has been conducted in people with $\mathrm{MCl}$ to determine whether biomarkers, such as $18 \mathrm{~F}$-flutemetamol for $A \beta$, increase the risk of developing dementia over time. The evidence for this is uncertain. For this and other reasons, the NIAAA in the USA established two different criteria for $\mathrm{MCl}$. Firstly, they established the Core Clinical Criteria for use in all clinical settings, without use of biomarkers, and characterised by concerns regarding a change in cognition with impairment in one or more cognitive domains with preservation of independence in functional abilities, therefore no dementia. Secondly, they established the Clinical Research Criteria, which incorporate the use of biomarkers, such as PET amyloid scans, intended for use exclusively in research settings, including academic centres and clinical trials. This will help determine whether positive scans increase the likelihood of progression from MCI to clinical dementia (Albert 2011). Lastly, it is hoped that people with $\mathrm{MCl}$ and positive scans will 'enrich' clinical trials, and more people who will progress to dementia in a shorter time will be included to allow more efficient studies of treatments and prevention strategies of ADD (CMS 2013).

An assumption for some researchers, and one on which this systematic review (SR) is predicated, is that if a person has both $\mathrm{MCl}$ and the pathology of Alzheimer's disease and develops clinical ADD subsequently, then the cause of the initial $\mathrm{MCl}$ and of the ADD was the Alzheimer's pathology. Our approach is an example of assessing diagnostic test accuracy (DTA) using delayed verification of diagnosis. Instead of the reference standard being based on pathology, it is based on a clinical standard and the progression from $\mathrm{MCl}$ to $\mathrm{ADD}$, or any other form of non-ADD, or any dementia. Although, for the reasons stated above, a degree of unreliability has been introduced, defining progression has the advantage of being based on what matters most to people with $\mathrm{MCl}$, their families, and clinicians involved in their care.

18 F-flutemetamol PET scan is considered the diagnostic marker of interest, and in this SR we assessed the DTA of $18 \mathrm{~F}$-flutemetamol A $\beta$ binding in the brain and progression of the following:

- From $\mathrm{MCl}$ to ADD.

- From $\mathrm{MCl}$ to any other form of non-ADD.

- From MCl to any form of dementia

This SR belongs to a series of SRs regarding PET biomarkers for A $\beta$, including ${ }^{18} \mathrm{~F}$-florbetaben and $18 \mathrm{~F}$-florbetapir (Martínez 2016).

\section{Target condition being diagnosed}

This SR assessed the following three target conditions.

- $\mathrm{ADD}$ (progression from $\mathrm{MCl}$ to $\mathrm{ADD}$ ).

- Any other form of dementia (progression from $\mathrm{MCl}$ to any other form of non-ADD).

- Any form of dementia (progression from $\mathrm{MCl}$ to any form of dementia).
We compared the index test results obtained at baseline with the results of the reference standards obtained at follow-up (delayed verification).

\section{Index test(s)}

The $18 \mathrm{~F}$-flutemetamol scan is an index test for the detection of $A \beta$ deposition in the brain region of interest (ROI). The ROI is a selected brain area that physicians create for further study in various anatomical areas of the brain. ${ }^{18} \mathrm{~F}$-flutemetamol is a molecular biomarker, described as follows.

- 18F-flutemetamol $A \beta$ is described as 6-benzothiazolol, 2-[3$\left[{ }^{18} \mathrm{~F}\right]$ fluoro-4-(methylamino)phenyl], and is also referred to as 18F-3'-F-6-OH-BTA1, 18F-GE067, AH110690 (Koole 2009; Nelissen 2009).

- $18 \mathrm{~F}$-flutemetamol has been evaluated in people with ADD, $\mathrm{MCl}$, and healthy controls in a clinical field in order to identify a valid, simple, and reliable PET quantitation method for the routine measure of brain amyloid retention in vivo (Vandenberghe 2010).

\section{Image Interpretation}

Both the FDA and EMA have described the criteria for $18 \mathrm{~F}$ flutemetamol for A $\beta$ positivity (EMA 2014; FDA 2014).

18 F-flutemetamol diagnosis is by PET image assessment, and is defined as positive if analysis shows the following.

- At least one cortical region (frontal lobes, posterior cingulate and precuneus, lateral temporal lobes, inferolateral parietal lobes, striatum) with reduction or loss of the normally distinct grey-white matter contrast. These scans have one or more regions with increased cortical grey matter signal (above 50\% to $60 \%$ peak intensity) or reduced (or absent) grey-white matter contrast (white matter sulcal pattern is less distinct), or both.

- A positive scan may have one or more regions in which grey matter radioactivity is as intense or exceeds the intensity in adjacent white matter.

Readers trained in PET images with the $18 \mathrm{~F}$-flutemetamol should interpret the A $\beta$ PET images made with this ligand (EMA 2014; FDA 2014).

Before the FDA and EMA described the criteria for 18 F-flutemetamol scan positivity, the diagnosis of dementia was made using different thresholds. Therefore, we planned to use the FDA or EMA criteria applied in each included study to classify participants as either testpositive or test-negative, or, alternatively, if $18 \mathrm{~F}$-flutemetamol $A \beta$ uptake and retention exceeded a certain threshold.

We considered the measurement of the $18 \mathrm{~F}$-flutemetamol retention (retention ratio): distribution volume ratio (DVR), standardised uptake value ratio (SUVR), or other ratios. DVR refers to the ratio of the $18 \mathrm{~F}$-flutemetamol distribution volume in the selected area (ROI) to the distribution volume in the reference area. SUVR is the ratio of the $18 \mathrm{~F}$-flutemetamol ligand standardised uptake value in the selected area (ROI) to the standardised uptake value in the reference area.

The unit of analysis of our SR was the participant. We did not include studies that analysed multiple ROIs per participant.

18F PET with flutemetamol for the early diagnosis of Alzheimer's disease dementia and other dementias in people with mild cognitive 
Image analysis: not prespecified (e.g. Statistical Parametric Mapping (SPM) or other image analysis techniques).

\section{Administration Instructions and Recommended Dosing}

- Time between 18 F-flutemetamol injection and PET acquisition: images should be acquired in 20 minutes starting from 90 minutes after intravenous administration (EMA 2014; FDA 2014).

- Injection dose: the recommended dose for $18 \mathrm{~F}$-flutemetamol $A \beta$ PET is $185 \mathrm{MBq}(5.0 \mathrm{mCi})$ administered as a single slow intravenous bolus (EMA 2014; FDA 2014).

Although it was inevitable that included studies had used different imaging protocols, readers' expertise, and varied parameters, the amyloid PET data in these included studies should be technically adequate and acquired at a fully qualified and certified facility.

\section{Clinical pathway}

At this time, the clinical evaluation often has similarities between different countries (Cordella 2013; NICE 2006). It often starts with people experiencing memory complaints detected by themselves or their relatives. Frequently, general practitioners or family physicians are consulted, and they often conduct a medical evaluation using a screening test for cognitive impairment. Whenever this screening test is positive, they complete an assessment with a clinical evaluation conducted with laboratory studies that can rule out a secondary cause of cognitive impairment (e.g. hypothyroidism, renal failure, liver failure, vitamin B12 or folate deficiency, and others). In addition, these people are then referred to medical specialists in cognitive disorders (preferably a geriatrician, psychiatrist, or neurologist) in a secondary centre or directly to memory clinics where further clinical assessment, laboratory studies, and cerebral image studies are conducted to confirm the dementia diagnosis.

People with dementia, or their relatives, often directly consult these specialists or specialised memory clinics in the study of cognitive disorders. Therefore, the performance of the diagnostic tests will probably vary according to whether it is a primary consultation or referral from primary to specialist care, or if the people have different clinical stages of the disease $(\mathrm{MCl}$, mild, moderate, or severe dementia). Due to these differing pathways, the use of 18 F-flutemetamol PET ligand for $A \beta$ is mainly used in specialist consultations and memory clinics as an addition to clinical evaluation or other tests, helping in a clinical setting to discard a diagnosis of Alzheimer's dementia with a negative scan in a person with clinical dementia and doubts about the aetiology (e.g. FTD versus ADD). Otherwise, it might be used solely in the research field in people with $\mathrm{MCl}$ for the enrichment of clinical trials, for example, enrolling people with $\mathrm{MCI}$ and a positive PET scan to study preventive interventions before people develop dementia.

However, in some memory clinics the $18 \mathrm{~F}$-flutemetamol PET is used for clinical purposes in people with persistent or progressive unexplained $\mathrm{MCl}$ adopting the Johnson criteria (Johnson 2013), criteria without sufficient evidence. Therefore, if the ${ }^{18} \mathrm{~F}$-flutemetamol PET is positive in a person with $\mathrm{MCl}$, this positivity is considered as one of the core histopathological findings of Alzheimer's disease. The person will thus be catalogued as a patient with prodromal Alzheimer's disease or $\mathrm{MCl}$ due to Alzheimer's disease.

\section{Alternative test(s)}

Currently there are no standard practice tests available for the clinical diagnosis of Alzheimer's disease dementia. Below, we have listed the alternative tests that we have excluded from this SR. The Cochrane Dementia and Cognitive Improvement Group is in the process of conducting a series of DTA SRs of biomarkers and scales (see list below).

- 18F PET ligands for $\mathrm{A} \beta$ (18F-florbetapir, 18F-florbetaben) (Martínez 2016).

- 18F-FDG-PET (PET F-fluorodeoxyglucose) (Smailagic 2015).

- 11C-PIB-PET (PET-Pittsburgh compound B) (Zhang 2014).

- Cerebrospinal fluid (CSF) analysis of A $\beta$ and tau (Kokkinou 2014; Ritchie 2013; Ritchie 2014).

- Structural magnetic resonance imaging (sMRI) (Filippini 2012).

- Neuropsychological tests (Mini-Mental State Examination (MMSE); MiniCOG; Montreal Cognitive Assessment (MoCA) (Arevalo-Rodriguez 2015; Chan 2014; Creavin 2016; Davis 2015; Fage 2015; Seitz 2014).

- Informant interviews (Informant Questionnaire on Cognitive Decline in the Elderly (IQCODE); AD8) (Harrison 2014; Hendry 2014; Lees 2014; Harrison 2015; Quinn 2014).

- APOE- $€ 4$ (Elias-Sonnenschein 2014a; Elias-Sonnenschein 2014b; Elias-Sonnenschein 2014c).

- Single-photon emission computed tomography (SPECT) brain imaging (Archer 2015; McCleery 2015).

\section{Rationale}

Accurate and early diagnosis of Alzheimer's disease is crucial for planning in healthcare systems, because the costs of dementia are currently at least $1 \%$ of the world's GNP (WHO 2012).

18 F-flutemetamol is approved for use in the clinical field mainly in people who are diagnosed clinically with dementia of uncertain aetiology, in which case diagnosis of ADD can be discarded if the test is negative. Even though $18 \mathrm{~F}$-flutemetamol is not approved for this purpose, this biomarker test is currently being used in the research field to search for the accurate identification of people with $\mathrm{MCl}$ who would progress to ADD or other forms of dementia. Amyloid $\beta$ tracers by PET have been included in newly diagnostic criteria in the study in people with $\mathrm{MCl}$ (Albert 2011; Dubois 2014). However, some uncertainties exist about the generalisability of the DTA results in clinical settings, especially in older people (Richard 2012).

It is currently believed that if the health system can identify which people are at high risk of progressing from $\mathrm{MCl}$ to dementia, it can focus on improving opportunities for appropriate contingency planning for them. Proper recognition of the disease may also help prevent inappropriate and potentially harmful admissions to hospital or institutional care (NAO 2007), and enable the development of new treatments designed to delay or prevent progression to more debilitating stages of the disease. Additionally, this may demonstrate a real clinical benefit for people and caregivers, and will reduce health system costs.

This SR assesses the DTA with ${ }^{18} \mathrm{~F}$-flutemetamol A $\beta$ PET in people with $\mathrm{MCl}$. 


\section{O B JECTIVES}

To determine the diagnostic test accuracy (DTA) of $18 \mathrm{~F}$ flutemetamol as the index test for detecting people with mild cognitive impairment $(\mathrm{MCl})$ at time of performing the test who would clinically progress to Alzheimer's disease dementia (ADD), or other forms of non-ADD, or any form of dementia at follow-up.

\section{Secondary objectives}

To investigate the heterogeneity of the DTA in the included studies, by evaluating the spectrum of people, referral centres, clinical criteria of $\mathrm{MCl}, 18 \mathrm{~F}$-flutemetamol techniques, reference standards used, duration of follow-up, aspects of study quality, and conflicts of interest.

\section{METHODS}

\section{Criteria for considering studies for this review}

\section{Types of studies}

We included longitudinal studies that had prospectively defined cohorts with any accepted definition of mild cognitive impairment $(\mathrm{MCl})$, as outlined below, at time of performing the $18 \mathrm{~F}$ flutemetamol $A \beta$ scan and a reference standard (see Index tests and Reference standards below). We obtained the results at the follow-up of the studies. These studies had to employ delayed verification of progression to dementia and were sometimes labelled as 'delayed verification cross sectional studies' (Bossuyt 2008; Knottnerus 2002). We included case-control studies when they incorporate a delayed verification design. This occurred in the context of a cohort study, so these studies were invariably diagnostic-nested case-control studies.

\section{Participants}

Participants recruited and clinically classified as having $\mathrm{MCl}$ at time of performing the test were eligible for inclusion. We established the diagnosis of $\mathrm{MCl}$ using the Petersen criteria or revised Petersen criteria (Petersen 1999; Petersen 2004; Winblad 2004), the criteria included in Matthews study (Matthews 2008), CDR $=0.5$ (CDR structured interviews collects information from both the collateral source and the subject regarding memory, orientation, judgment and problem solving, community affairs, home and hobbies, and personal care, where the range of possible scores varies from none $=0$ point to severe $=3$ points) (Morris 1993), the National Institute on Aging-Alzheimer's Association (NIA-AA) core clinical criteria (Albert 2011), or a combination.

We excluded studies that included people with $\mathrm{MCl}$ possibly caused by any of the following:

- Current or a history of alcohol or drug abuse.

- Central nervous system (CNS) trauma (e.g. subdural hematoma), tumour, or infection.

- Other neurological conditions (e.g. Parkinson's or Huntington's diseases). Regarding Parkinson's disease, many of the studies specifically excluded people with Parkison's disease from the group with mild cognitive impairment. This specific group of people is complex in both regards to defining neuropathology and in determination of functional decline. For these reasons this group of people needs to be addressed in specific studies

\section{Index tests}

The index test of this SR was ${ }^{18} \mathrm{~F}$-flutemetamol biomarker test. We used the criteria and cut-off values for test positivity, as reported in the included studies. We considered positivity for ${ }^{18} \mathrm{~F}$-flutemetamol $\mathrm{A} \beta$ scan uptake and retention exceeding a certain threshold.

\section{Target conditions}

Three target conditions were included in this SR:

- Alzheimer's disease dementia (ADD) (progression from $\mathrm{MCl}$ to ADD).

- Any other forms of dementia (progression from MCI to any other forms of non-ADD).

- Any form of dementia (progression from $\mathrm{MCl}$ to any form of dementia).

\section{Reference standards}

The reference standard was the progression to the target conditions evaluated by a physician with expertise in the dementia field (preferably a geriatrician, psychiatrist, or neurologist). For the purpose of this SR, we accepted several definitions of ADD. We included studies that applied the National Institute of Neurological and Communicative Disorders and Stroke and the Alzheimer's Disease and Related Disorders Association (NINCDSADRDA) criteria (McKhann 1984), the Diagnostic and Statistical Manual of Mental Disorders (DSM) criteria (APA 1987; APA 1994), and the International Classification of Diseases (ICD) (ICD-10) criteria for ADD. Notably, different iterations of these standards may not be directly comparable over time (e.g. APA 1987 versus APA 1994). Moreover, the validity of the diagnoses may vary with the degree or manner in which the criteria have been operationalised (e.g. individual clinician versus algorithm versus consensus determination). We considered all these issues when we interpreted the results.

Similarly, we accepted differing clinical definitions of other dementias. For Lewy Body Dementia the reference standard is the McKeith criteria (McKeith 1996; McKeith 2005); for frontotemporal dementia the Lund criteria (Boxer 2005; Brun 1994; Neary 1998), the DSM criteria (APA 1987; APA 1994), the ICD criteria (ICD-10), or the International Behavioural Variant FTD Criteria Consortium (Rascovsky 2011); and, for vascular dementia, the National Institute of Neurological Disorders and Stroke and Association Internationale pour la Recherché et l'Enseignement en Neurosciences (NINDS-AIREN) criteria (Román 1993), the DSM criteria (APA 1987; APA 1994), or the ICD criteria (ICD-10).

The time interval in which the progression from $\mathrm{MCl}$ to $\mathrm{ADD}$ (or other forms of dementia) occurs is very important. We used one year as the minimum period of delay in the verification of the diagnosis (the time between the assessment at which a diagnosis of $\mathrm{MCl}$ is made and the assessment at which the diagnosis of dementia is made).

\section{Search methods for identification of studies}

\section{Electronic searches}

We searched MEDLINE (Ovid SP) from 1946 to May 2017; Embase (Ovid SP) from 1974 to May 2017; PsycINFO (Ovid SP) from 1806 to May 2017; BIOSIS Citation Index (Thomson Reuters Web of Science) from 1922 to May 2017; Web of Science Core Collection, including the Science Citation Index (Thomson Reuters Web of Science) and the Conference Proceedings Citation Index (Thomson Reuters

18F PET with flutemetamol for the early diagnosis of Alzheimer's disease dementia and other dementias in people with mild cognitive 10 impairment (MCI) (Review)

Copyright (c) 2017 The Cochrane Collaboration. Published by John Wiley \& Sons, Ltd. 
Web of Science) from 1946 to May 2017; LILACS (Bireme); CINAHL (EBSCOhost) from 1980 to May 2017; ClinicalTrials.gov (https:// clinicaltrials.gov); and the World Health Organization International Clinical Trials Registry Platform (WHO ICTRP) (http://www.who.int/ ictrp/search/en/). We also searched ALOIS, the Cochrane Dementia \& Cognitive Improvement Group's specialized register of dementia studies (http://www.medicine.ox.ac.uk/alois/).

We used two approaches in designing the search. One focused solely on the specifically named index test (including a range of synonyms) and the second, run in parallel covered a more general search, linking broader terms for the index test. It focused on terms describing its diagnostic use and terms for the target condition to try to capture the more difficult to locate studies of a more general nature, where these particular radioligands were included in diagnostic accuracy research but not named specifically in the parts of the electronic bibliographic record that are searchable and therefore would be missed.

See Appendix 2 for details of the sources and search strategies that we used. No language or date restrictions were applied to the electronic searches.

\section{Searching other resources}

We examined the reference lists of all relevant studies for additional studies. We also searched the Database of Abstracts of Reviews of Effects (DARE) via the Cochrane Library (www.cochranelibrary.com)), the National Institute for Health Research - Health Technology Assessment Database (NIHR-HTA) (via the Cochrane Library: www.cochranelibrary.com), the Aggressive Research Intelligence Facility (ARIF) database (www.arif.bham.ac.uk) for other related systematic diagnostic accuracy reviews, and the International Federation of Clinical Chemistry and Laboratory Medicine Committee for Evidence-based Laboratory Medicine database (C-EBLM) (http://www.ifcc.org/ifcc-education-division/ emd-committees/c-eblm/evidence-based-laboratory-medicine-ceblm-base).

We checked the reference lists of any relevant studies and SRs, and performed citation tracking using the Science Citation Index to identify any additional relevant studies.

\section{Data collection and analysis}

\section{Selection of studies}

Two review authors (GM, RV) independently screened the retrieved titles and abstracts for potentially eligible studies. A third review author (PF) resolved any disagreements between the two review authors. The two review authors (GM, RV) then independently assessed the full-text articles of the selected studies with the inclusion criteria. They resolved any disagreements through discussion or, where necessary, consulted a third review author (PF) who acted as an arbitrator. When a study did not present all relevant data for creating $2 \times 2$ table, we contacted the study authors directly to request further information. When more than one article presented data on the same population, we included the primary article, which was the article with the largest number of people or with the most informative data (e.g. longest time of follow-up in the primary outcome).

\section{Data extraction and management}

We planned to extract the following data regarding the study characteristics.

- Bibliographic details of primary paper:

* author, title of study, year, and journal.

- Basic clinical and demographic details:

* number of participants;

* clinical diagnosis;

* $\mathrm{MCl}$ clinical criteria;

* age;

* gender;

* sources of referral;

* participant recruitment;

* sampling procedures.

- Details of the index test:

* method of the 18 F-flutemetamol test administration, including those who administered the test;

* thresholds used to define positive and negative test;

* other technical aspects as seemed relevant to the review, e.g. brain areas.

- Details of the reference standard:

* definition of ADD and other dementias used in reference standard;

* duration of follow-up from time of the index test performed to defining ADD and other dementias by the reference standard: one year to less than two years; two years to less than four years; and four years or more. If participants had been followed for varied amounts of time we recorded a mean follow-up period for each included study. If possible, we grouped those data into minimum, maximum, and median follow-up periods, to enable subgroup analyses;

* prevalence or proportion of population developing ADD and other dementias, with severity if described.

We created $2 \times 2$ tables (cross-relating index test results of the reference standards) as shown in Appendix 3. For each included study, we recorded the number of participants lost to followup. We also extracted data necessary for the quality assessment, as defined below. Two review authors (GM, RV) independently performed data extraction. We resolved any disagreements regarding data extraction by discussion, or consulting a third review author (PF), if it was necessary.

\section{Assessment of methodological quality}

We assessed the methodological quality of the included studies using the Quality Assessment of Diagnostic Accuracy Studies 2 tool (QUADAS-2) (Whiting 2011), as recommended by Cochrane (Davis 2013). This tool is comprised of four domains: patient selection, index test, reference standard, and patient flow.

Two review authors (GM, RV), who were blinded to each other's scores, independently performed the QUADAS-2 assessment. We resolved any disagreements by discussion or, if necessary, consulted a third review author (PF) who acted as an arbitrator. We assessed each domain in terms of risk of bias, and also considered the first three domains in terms of applicability concerns. In Appendix 4, we have detailed the components of each of these domains and provided a rubric that shows how we made

18F PET with flutemetamol for the early diagnosis of Alzheimer's disease dementia and other dementias in people with mild cognitive 11 impairment (MCI) (Review)

Copyright (c) 2017 The Cochrane Collaboration. Published by John Wiley \& Sons, Ltd. 
judgements concerning risk of bias. Key areas important to quality assessment were participant selection, blinding, and missing data.

We included three additional signalling questions on our checklist.

- Was the PET scan interpretation done by a trained reader physician? (We included this under the 'Index test' domain.)

- Was there a clear definition of a positive result? (We included this under the 'Index test' domain.)

- Was the study free of commercial funding? (We included this under the 'flow and timing' domain.)

We included the item pertaining to the PET scan interpretation and the definition of positive results to take into account the subjective nature of the $18 \mathrm{~F}$-flutemetamol $A \beta$ scan image interpretation, which may be based on a variety of different criteria, such as extensive clinical experience, different standardised uptake values (SUV), different morphological features, or a combination of the aforementioned. We included the third additional item in order to record any potential bias resulting from commercial interest in the results due to the potential risk by the manufacturing company leading to more favourable results and conclusions than sponsorship by other sources (Lundh 2017).

We did not use QUADAS-2 data to form a summary quality score. We produced a narrative summary that described each included study as at high, low, or unclear risk of bias, as well as concerns regarding applicability, which we have described in Appendix 5.

\section{Statistical analysis and data synthesis}

We applied the DTA framework for the analysis of a single test and extracted the data from each included study into a $2 \times 2$ table, showing the binary test results cross-classified with the binary reference standard, and we ignored any censoring that might have occurred. We acknowledge that such a reduction in the data may represent a significant oversimplification. We used data from the $2 \times 2$ tables abstracted from the included studies: true positive (TP), false negative (FN), false positive (FP), true negative (TN), and entered these into Review Manager (RevMan) (Review Manager 2014) to calculate the sensitivities, specificities, and their $95 \%$ confidence intervals. We also presented individual study results graphically by plotting estimates of sensitivities and specificities in both a forest plot. If an individual included study published more than one threshold, we presented the graphical findings for all reported thresholds.

We planned to segment analyses into separate follow-up mean periods for the delay in verification: one year to less than two years; two to less than four years; and greater than four years. In this we planned to clearly note where the same included studies contributed to the analysis for more than one reference standard follow-up interval.

However, due to lack of data, we conducted no meta-analyses. However, we prepared a 'summary of findings table'.

\section{Investigations of heterogeneity}

We were able to include only two studies, therefore issues of heterogeneity did not arise.

\section{Sensitivity analyses}

We found insufficient data to conduct any sensitivity analyses.

\section{Assessment of reporting bias}

We did not investigate reporting bias.

\section{RE S U L T S}

\section{Results of the search}

The total number of records identified for this SR was 1333 . The PRISMA diagram (Figure 1) shows the selection of records through the screening and selection processes. In total, we assessed 81 studies ( 23 full-text papers, 22 conference publications, 11 registered studies in clinicaltrials.gov, and 25 registered studies in WHO ICTRP) for eligibility in the full-text screening. We excluded 72 studies. Ten studies were multiple publications or duplicated and 4 studies did not have extractable data for constructing 2 $\times 2$ tables, and we received no reply when we contacted the authors (Goukasian 2015; Rowe 2015a; Rowe 2015b; Rowe 2015c) (Characteristics of excluded studies). We excluded the remaining 58 studies because they did not meet the inclusion criteria: i) not a longitudinal study $(\mathrm{n}=23)$; ii) no $\mathrm{MCl}$ participants at time of performing the test $(n=21)$; iii) index test not a 18 F-flutemetamol PET scan $(n=4)$; iv) discussion or review paper $(n=6)$; v) wrong outcomes or design $(n=4)$. We included two studies and identified seven references as ongoing studies. 
Figure 1. Flow diagram.

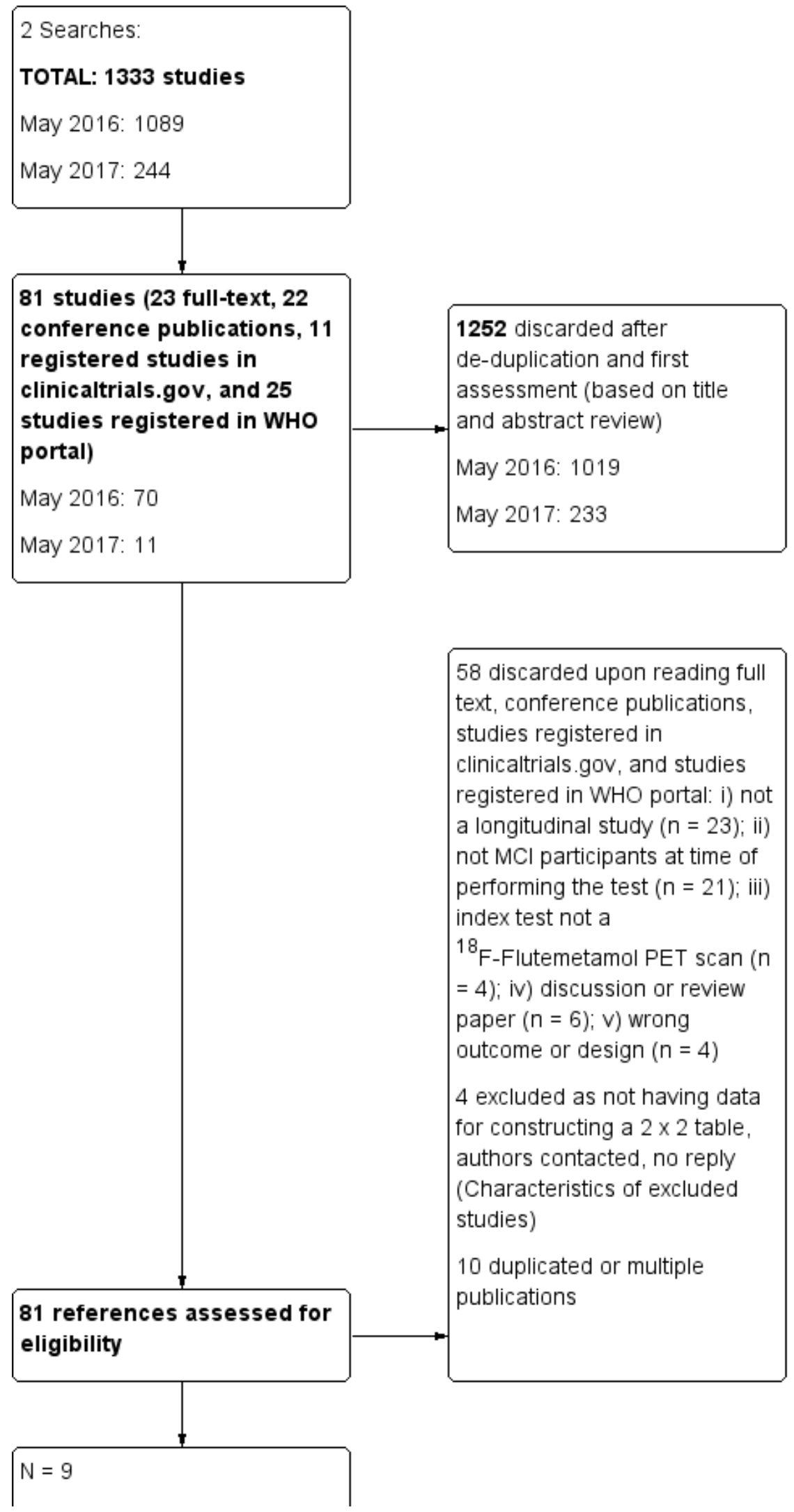

18F PET with flutemetamol for the early diagnosis of Alzheimer's disease dementia and other dementias in people with mild cognitive 
Figure 1. (Continued)

$$
\mid \begin{aligned}
& N=9 \\
& 2 \text { included studies } \\
& 7 \text { ongoing studies }
\end{aligned}
$$

\section{Included studies}

\section{See Characteristics of included studies.}

Thurfjell 2012 refers to a study with baseline data that had been published 2 years earlier with $\mathrm{ADD}, \mathrm{MCl}$ and healthy controls (HC) participants. $\mathrm{MCl}$ participants were recruited from secondary care (7 memory clinics). Participants were recruited as part of a study to evaluate the $18 \mathrm{~F}$-flutemetamol PET scan in people with ADD ( $\mathrm{n}=27)$, amnestic $\mathrm{MCl}(\mathrm{n}=20)$ and healthy controls $(n=20)$ as a cross-sectional study to determine the efficacy of blinded visual assessment of images of $18 \mathrm{~F}$-flutemetamol uptake for separating subjects with clinically probable ADD from healthy controls, the SUVRs of subjects with probable $\mathrm{ADD}$ and $\mathrm{HC}$, the concordance between (11)C-labelled Pittsburgh Compound-B ((11)C-PIB) and $18 \mathrm{~F}$-flutemetamol scans, regarding visual assessment and quantitative SUVR in ADD and $\mathrm{MCl}$ participants, and the assignation of a raised or low amyloid group category through visual or quantitative assessment in $\mathrm{MCl}$ participants (Vandenberghe 2010).

The study of Thurfjell 2012 included $20 \mathrm{MCl}$ participants with a follow-up of two years to evaluate the progression from amnestic $\mathrm{MCI}$ to probable ADD according to their ${ }^{18} \mathrm{~F}$-flutemetamol status as positive or negative, using a SUVR $>1.5$ (Thurfjell 2012). The SUVR established in the previous study with ADD and HC participants was 1.56. The other objective of this study was to compute the hippocampus volume from MRI and investigate its accuracy performance alone and combined with the 18 F-flutemetamol PET scan at follow-up. MCl participants fulfilled Petersen 1999 criteria for amnestic $\mathrm{MCl}, 11$ were male, they had a mean age of $72.7 \pm 7.09$ years, with $14.4 \pm 2.97$ mean years of education, and their mean MMSE was $28.0 \pm 0.94$ points.

Of the 20 participants, 9 (45\%) developed Alzheimer's dementia. One participant (5\%) was reported as lost to follow-up without further information about the cause.

The reference standard was not explicitly stated, although NINCDSADRDA criteria for ADD (McKhann 1984) and APA 1994 were baseline diagnostic criteria in the Vandenberghe study (Vandenberghe 2010).

Potential conflicts of interest were noted. Financial support for the baseline study (Vandenberghe 2010) was from the manufacturer of $18 \mathrm{~F}$-flutemetamol tracer and two authors were employees of this company.

NCT01028053 refers to an international and multicentric study in the United States and Europe (also known as EUCTR2009-010227-62-GB in Europe) and with a common sponsor's protocol code number, GE-067-005.
The main objective of this study was to evaluate the 'hazard ratio by PET scan readers for conversion to probable Alzheimer's disease based on visual image Interpretation' in amnestic $\mathrm{MCl}$ participants with normal and abnormal patterns of $18 \mathrm{~F}$-flutemetamol uptake, based on the visual assessment of a $18 \mathrm{~F}$-flutemetamol PET scan. This unpublished study had no information regarding the participants' recruitment. There were 230 planned evaluable participants. The participants were 60 years old or older (US inclusion criteria in clinicaltrials.gov) or over 55 years old (Europe in EUDRACT), they met the Petersen criteria for amnestic $\mathrm{MCl}$ (not provided which of the different Petersen criteria published were used), had a score of less than or equal to 4 on the Modified Hachinski Ischemic Scale, a MMSE score of 24 to 30, and a non-contrast MRI examination as part of the screening visit that excluded amnestic $\mathrm{MCl}$ arising from structural causes, and they had no significant neurologic disease other than suspected amnestic $\mathrm{MCl}$. The mean age was $71.1(+8.62)$ years, 63 participants were less than 65 years, and 118 were women.

Participants were assessed clinically on-site every six months until progression to probable ADD (as determined by an independent Clinical Adjudication Committee (CAC)); or completion of 36 months of follow-up, whichever came first. Clinical assessments were performed by a trained on-site clinician who collected the results of a battery of tests, the National Institute of Neurological and Communicative Disorders and Stroke-Alzheimer's Disease and Related Disorders Association (NINCDS-ADRDA) diagnostic criteria for probable ADD, and clinical assessment; this clinician was blinded to the subjects' PET images and interpretations until the study was complete. The follow-up data were regularly submitted to the CAC (which consisted of four experts in the diagnosis of memory disorders), which determined whether or not the subject had converted to probable ADD. The CAC reviewed all study data (excluding the investigator's progression assessment, the ${ }^{18} \mathrm{~F}$ flutemetamol PET scan results and any other amyloid imaging data) for each subject to determine whether or not the subject had converted to probable ADD. The decision rules to be used in defining a progression to probable ADD were established by the CAC before reviewing any subject's data.

The study analysed 224 participants of the original 232 participants at 36 months of follow-up, because 8 participants withdrew before the first assessment at the follow-up.

Potential conflicts of interest were noted. Total financial support was provided from the manufacturer of $18 \mathrm{~F}$-flutemetamol tracer.

\section{Excluded studies}

We excluded four studies since they did not meet the inclusion criteria for participants, index test, or target condition (Characteristics of excluded studies). 
The Goukasian 2015 study was focused on neuropsychiatric symptoms with a probable follow-up in $38 \mathrm{MCl}$ participants with a SUVR $>1.27$ for brain amyloidosis. In Rowe 2015a, there were 59 participants with $\mathrm{MCl}$ at the time of performing the test and at the end of 18 months of follow-up, there were 16 participants to be evaluated. In Rowe 2015b, there were $50 \mathrm{MCl}$ participants and at the end of 18 months of follow-up, there were seven participants to be evaluated. In Rowe $2015 \mathrm{c}$, there were 17 participants with $\mathrm{MCl}$ evaluated at 18 months of follow-up. All of these three studies were focused on change of $18 \mathrm{~F}$-flutemetamol PET scan retention over time and probably shared participants, so it is possible that these reports referred to the same study.

None of the authors answered our email inquiries for additional information.

\section{Ongoing studies}

We found two ongoing studies in clinicaltrials.gov. NCT02164643 is a study that focused on participants with different cognitive spectrums, from isolated cognitive complaints to $\mathrm{MCl}$ with a basal $18 \mathrm{~F}$-flutemetamol or $18 \mathrm{~F}$-florbetapir PET scan and the progression to a clinical dementia stage according to DSM-IV and NINCDSADRDA as reference standards for up 24 months follow-up. This study has been recruiting participants since July 2014 in France. The second study, NCT02196116, is focused on the amyloid load in three different participants in a cross-sectional study: controls, $\mathrm{MCl}$ without memory complaints, and $\mathrm{MCl}$ with memory complaints. However, they also considered a longer term clinical follow-up of study participants to investigate the prognosis value of amyloid load for improving the prediction of cognitive decline and disease progression. No further information about the follow-up was detailed.

We found five ongoing studies in the WHO ICTRP register. EUCTR2011-001756-12-BE is a study focused on cognitively healthy older people and $\mathrm{MCl}$ participants. The main objective is to evaluate, with a multimarker approach, the amnestic $\mathrm{MCl}$ participants by quantitative analysis of each biomarker by comparison to a normal database of recruited healthy volunteers and a clinical follow-up from one to three years with basal $18 \mathrm{~F}$-flutemetamol. No further details were provided regarding the participants, index test, and reference standard(s). This study has been ongoing since April 2012. The second study, EUCTR2011-006195-39-SE, is focused on $\mathrm{MCl}$ participants and the main objective is to examine the efficacy of raised $18 \mathrm{~F}$ flutemetamol brain uptake for differentiating people with mild cognitive impairment $(\mathrm{MCl})$, who subsequently will develop $A D D$, from people with $\mathrm{MCl}$ who will be cognitively stable or develop other dementias than ADD. No further details were provided regarding the participants, index test, and reference standard(s). This study has been ongoing since January 2012. The third study is JPRN-UMIN000019926, which is focused on preclinical Alzheimer's disease and $\mathrm{MCl}$ participants. Their main objective is to discriminate between $\mathrm{MCl}$ individuals at risk of development of Alzheimer dementia over an established follow-up of 36 months. The index test will be $11 \mathrm{C}$-PiB, ${ }^{18} \mathrm{~F}$-florbetapir, or ${ }^{18} \mathrm{~F}$-flutemetamol PET scan. No further details were given regarding index test, and reference standard(s). This study has been ongoing since January 2016. The fourth study, EUCTR2017-000094-36-SE, is focused on $\mathrm{MCl}$, dementia, and healthy elderly people and the main objective is to study the diagnostic accuracy of Tau PET $18 \mathrm{~F}-\mathrm{RO} 0958948$ and $18 \mathrm{~F}$-flutemetamol for identifying healthy elderly individuals and people with subjective or objective mild cognitive symptoms who are at high risk of subsequent development of ADD or other neurodegenerative disorders. The follow-up was not clearly stated. This study has been ongoing since March 2017. The fifth study, EUCTR2016-002635-15-NL, is focused on people aged 90 or older. The main objectives are to understand how clinical markers and biomarkers previously identified in younger and older ADD cohorts apply to the extremely old, to identify novel biomarkers linked with resilience to developing ADD in extremely old subjects and the generation of normative data for the oldest, and measure the concordance between amyloid pathology as assessed in CSF and by PET. This study has been ongoing since July 2016 .

\section{Methodological quality of included studies}

We assessed methodological quality using the QUADAS-2 tool (Whiting 2011). Review authors' judgements about each methodological quality item for each included study are presented in the Characteristics of included studies table. The overall methodological quality of the studies is summarised in Figure 2. 
Figure 2. Risk of bias and applicability concerns summary: review authors' judgements about each domain for each included study

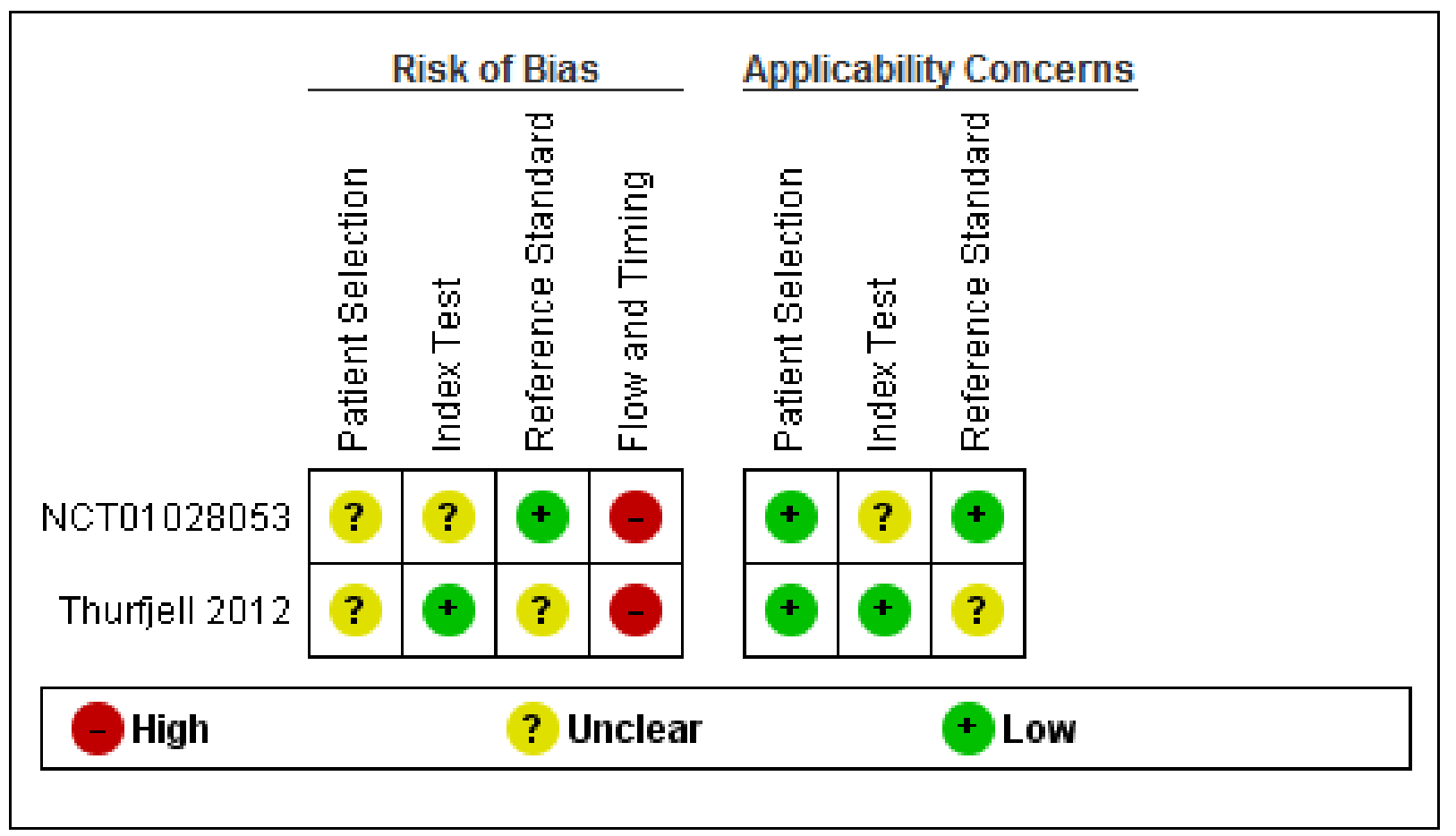

In the patient selection domain, we considered both studies (Thurfjell 2012; NCT01028053) to be at unclear risk of bias due to lack of reporting on sampling procedures and exclusion criteria. We stated that the included studies avoided a case-control design because we only considered data on performance of the index test to discriminate between people with $\mathrm{MCl}$ who converted to dementia and those who remained stable.

In the index test domain, we considered one study to have a low risk of bias and the other study to be at unclear risk of bias. The Thurfjell study had low risk of bias because the threshold used, according to Thurfjell 2012 references, was established in the previous study in ADD and HC participants as a SUVR $>1.56$ (Vandenberghe 2010), however, the SUVR used in this study was 1.5 and the index test results were interpreted without knowledge of the results of the reference standard. Regarding NCT01028053, the interpretation was made without knowledge of the reference standard, however the threshold was not clearly prespecified. In our two additional signalling questions, in the question on whether the index test was interpreted by a trained reader physician, this risk was unclear due to lack of information in the Thurfjell study, but no risk was identified in the NCT01028053 study. On the other hand, the other signalling question was rated as low risk in the Thurfjell study because there was a clear definition of a positive result, and unclear in NCT01028053 due to lack of information.

In the reference standard domain, we considered the Thurfjell study to have an unclear risk of bias because it was not reported if the clinicians conducting follow-up were aware of the initial $18 \mathrm{~F}$ flutemetamol result. We were not able to obtain the information about which reference standard was used, or how and by whom this reference standard was obtained, due to poor reporting (Thurfjell 2012). We judged NCT01028053, to be at a low risk of bias, because the reference standard used was NINCDS-ADRDA (McKhann 1984) and the CAC were blinded to the $18 \mathrm{~F}$-flutemetamol PET scan to established the reference standard.

In the flow and timing domain, we judged the Thurfjell study to have a high risk of bias because, in our additional signalling question, there were potential conflicts of interest due to financial support for the study (Vandenberghe 2010) and two authors of Thurfjell 2012 were employees from the manufacturer of the $18 \mathrm{~F}$-flutemetamol tracer. We judged the NCT01028053 study as having a high risk of bias due to possible conflict of interest due to financial support by the $18 \mathrm{~F}$-flutemetamol producer company.

For assessment of applicability, there was no concern that the included patients and setting, or the conduct and interpretation of the index test, did not match the review question; however, the target condition (as defined by the reference standard) was unclear due to lack to information about which reference standard(s) were applied and also the methodology used in the Thurfjell study (Thurfjell 2012). On the other hand, in NCT01028053, there was concern regarding the index test due to lack to information about the threshold and its definition.

\section{Findings}

The results of the included studies are summarised in Data table 1. Additionally, the summary of main results for the included studies are presented in the Summary of findings 1.

\section{F-flutemetamol for Alzheimer's disease dementia (ADD)}

NCT01028053 data on 224 of 232 eligible participants with amnestic $\mathrm{MCl}$ (diagnosed with Petersen criteria (not clear which of them were used), using NINCDS-ADRDA (McKhann 1984)) had a sensitivity of $64 \%(95 \% \mathrm{Cl} 53$ to 75$)$ and a specificity of $69 \%(95 \% \mathrm{Cl} 60$ to 76$)$ to

18F PET with flutemetamol for the early diagnosis of Alzheimer's disease dementia and other dementias in people with mild cognitive 16 impairment ( $\mathrm{MCl})$ (Review)

Copyright (C) 2017 The Cochrane Collaboration. Published by John Wiley \& Sons, Ltd. 
predict the progression from amnestic $\mathrm{MCI}$ to $\mathrm{ADD}$ at three years follow-up. Of 232 participants who were given an initial clinical diagnosis of amnestic $\mathrm{MCl}$, the study had data on 224 of them at the follow-up; 52 were true positive, 45 were false positive, 29 were false negative, and 98 were true negative (Figure 3 ).

\section{Figure 3. Forest plot of 18 F-flutemetamol.}

$\begin{array}{lrrrrrrrrr}\text { Study } & \text { TP } & \text { FP } & \text { FN } & \text { TN } & \text { Sensitivity } \mathbf{( 9 5 \%} \text { Cl) } & \text { Specificity (95\% Cl) } & \text { Sensitivity (95\% Cl) } & \text { Specificity (95\% Cl) } \\ \text { NCT01028053 } & 52 & 45 & 29 & 98 & 0.64[0.53,0.75] & 0.69[0.60,0.76] & & & \end{array}$

The criteria for 18 F-flutemetamol PET scan positivity was a visual assessment done by five blinded and trained readers, and they established the positivity or negativity of the PET scan according to the majority readings.

Thurfjell 2012 data on 19 of 20 eligible participants with amnestic MCl (diagnosed with Petersen criteria (Petersen 1999), using a nonspecified reference standard, probably NINCDS-ADRDA (McKhann 1984) and APA 1994) had a sensitivity of $89 \%(95 \% \mathrm{Cl} 52$ to 100$)$ and a specificity of $80 \%(95 \% \mathrm{Cl} 44$ to 97$)$ to predict the progression from amnestic $\mathrm{MCl}$ to $\mathrm{ADD}$ at two years follow-up. Of 20 participants who were given an initial clinical diagnosis of amnestic $\mathrm{MCl}$, the study had data on 19 of them at the follow-up; 8 were true positive, 2 were false positive, 1 was false negative and 8 were true negative (Figure 3).

The criterion for $18 \mathrm{~F}$-flutemetamol PET scan positivity was a quantitative threshold with a SUVR $>1.5$ and the measures of $18 \mathrm{~F}$ flutemetamol amyloid retention were:; lateral frontal cortex (FRO), lateral temporal cortex (LTC), lateral parietal cortex (PAR), anterior cingulate (ANC), occipital cortex (OCC), and pons (PON); a cerebellar $\mathrm{ROI}$ served as the reference region.

No data were available regarding the other two target conditions in this Cochrane review: progression from $\mathrm{MCl}$ to another form of dementia (non-ADD) or progression from $\mathrm{MCl}$ to any form of dementia.

\section{Investigation of heterogeneity}

The planned investigations were not possible due to the limited number of studies available for the analysis.

\section{Sensitivity analyses}

There were insufficient studies identified to permit any sensitivity analyses.

\section{DISCUSSION}

\section{Summary of main results}

The volume and quality of evidence regarding the DTA of $18 \mathrm{~F}$ flutemetamol for early diagnosis of ADD and other dementias in people with $\mathrm{MCl}$ is limited. We identified two studies in this SR. However, we were not able to construct a meta-analysis. We did not perform sensitivity analyses and were not able to analyse the heterogeneity.

The two included studies addressed the DTA of $18 \mathrm{~F}$-flutemetamol analysed quantitatively with a threshold of SUVR $>1.5$ (Thurfjell
2012) or by visual assessment (NCT01028053) for the prediction of progression from $\mathrm{MCl}$ to $\mathrm{ADD}$ at follow-up. The results are summarised in the 'Summary of findings' table (Summary of findings 1). The studies were evaluated as at high risk of bias mainly due to the potential conflict of interest because of the financial support of the company that manufactured the $18 \mathrm{~F}$ flutemetamol tracer. The study had no information about the progression to any form of dementia or any other form of dementia (non-ADD). Regarding our objectives, to determine the DTA of the $18 \mathrm{~F}$-flutemetamol PET scan for detecting participants with $\mathrm{MCl}$ at the time of performing the test who would clinically progress to ADD, or to other forms of dementia or any form of dementia at follow-up, the results were the following:

\section{F-flutemetamol PET scan for Alzheimer's disease dementia (ADD)}

Progression from $\mathrm{MCl}$ to $\mathrm{ADD}$ at three years of follow-up by visual assessment had a sensitivity of $64 \%(95 \% \mathrm{Cl} 53$ to 75$)$ and a specificity of $69 \%$ (95\% Cl 60 to 76 ) respectively $(n=224)$ (Figure 3 ).

Progression from $\mathrm{MCl}$ to $A D D$ at two years of follow-up by quantitative assessment by SUVR had a sensitivity of $89 \%(95 \% \mathrm{Cl}$ 52 to 100$)$ and a specificity of $80 \%(95 \% \mathrm{Cl} 44$ to 97$)$ respectively (n =19) (Figure 3).

The DTA of ${ }^{18} \mathrm{~F}$-flutemetamol includes a wide range of low-tomoderate and good sensitivity and specificity for predicting progression to ADD through visual or SUVR assessment evaluation at different follow-up. In other words, the low-to-moderate or good sensitivity could be affected by a relatively high false negative rate, admittedly from only one study. As with other amyloid tracers, $18 \mathrm{~F}$-flutemetamol probes the detection of amyloid plaques that are composed of insoluble $A \beta$ peptides (EMA 2014; FDA 2014). However, the soluble $A \beta$ oligomers play a central role in Alzheimer's pathogenesis in the amyloid hypothesis (Heyden 2013), with the possibility of producing false negatives. In addition, amyloid tracers are not be able to bind to the other histopathologic core of Alzheimer's disease - the neurofibrillary tangles (NFTs). There is evidence that suggests that plaques and tangles independently contribute to cognitive impairment over the clinical course of Alzheimer's disease (Serrano-Pozo 2013). Moreover, in another cohort study, the NFT formation might be either unrelated to amyloid plaques formation or a temporally distinct process, or both( Royall 2014 ). Another reason that could explain false negative results is that those with probable ADD may have multiple brain pathologies, most commonly Alzheimer's disease with macroscopic infarcts, followed by Alzheimer's disease with neocortical Lewy body disease, and, like ADD, MCI pathology could be heterogeneous (Schneider 2007; Schneider 2009).

18F PET with flutemetamol for the early diagnosis of Alzheimer's disease dementia and other dementias in people with mild cognitive 17 impairment ( $\mathrm{MCI}$ ) (Review)

Copyright @ 2017 The Cochrane Collaboration. Published by John Wiley \& Sons, Ltd. 
In addition, the low-to-moderate or good specificity could be affected by a high false positive rate. A positive $18 \mathrm{~F}$-flutemetamol PET scan for $A \beta$, has been found in other neurological conditions. It was positive in pure vascular dementia and Lewy body dementia cases confirmed by autopsy (Thal 2015). On the other hand, in other amyloid biomarkers like PET PiB, and closer to the $18 \mathrm{~F}$ flutemetamol chemical composition, the false positive rate could be explained because it has affinity to amyloid in vessel walls, in particular to cerebral amyloid angiopathy (CAA) (Zhang 2014). We would think that the pathological diagnosis of some people with clinical probable ADD may be vascular dementia secondary to CAA and some $\mathrm{MCl}$ participants may have vascular $\mathrm{MCl}$ due to CAA. The other important option for a high false positive rate is that in many people without cognitive impairment it is possible to find $A \beta$ deposits at their autopsies (Gelber 2012), generating some doubts about the real pathophysiological relevance of the $A \beta$ hypothesis in Alzheimer's disease.

Another important factor to be considered in predicting the progression to $A D D$ and the number of false positives is the duration of follow-up, because the reported progression rate of $\mathrm{MCI}$ to ADD is between $8 \%$ and $16 \%$ per year (Mitchell 2009). Therefore, a high percentage of people with $\mathrm{MCl}$ at the time of performing the test would progress to Alzheimer's disease if we had included a longer follow-up period, and this would affect the predictive DTA of the $18 \mathrm{~F}$-flutemetamol PET scan. However, the progression rate at two years was $47.4 \%$ and $36.2 \%$ at three years of follow-up in the included studies. The latest was found in a systematic review with PiB PET where the data were separated into short follow-up and longer than two years of follow-up. They included five studies with 102 participants in total, with a specificity between $58 \%$ to $100 \%$ (Ma 2014). However, in our study, the follow-up time and percentage of progression were discordant; the progression rate at two years was $47.4 \%$ and $36.2 \%$ at three years of follow-up in the included studies. This difference is probably explained by the setting of recruitment or demographic or $\mathrm{MCl}$ characteristics and possibly other underlying factors that were affecting the data (Thurfjell 2012; NCT01028053). As a consequence, due to the lack of data, we were not able to investigate the effect of the follow-up on the progression rate from $\mathrm{MCl}$ to $\mathrm{ADD}$ or any form of dementia.

On the other hand, $\mathrm{MCl}$ subtypes have been related to progression to dementia. A large longitudinal study with $550 \mathrm{MCl}$ participants indicated that the $\mathrm{MCl}$ subtype, presence of storage memory impairment, multiple domain condition, and presence of APOE $\epsilon 4$ allele increased the risk of progression to dementia. Multivariate survival and Kaplan-Meier analyses showed that amnestic MCl with storage memory impairment had the most and closest risk of progression to dementia (Espinosa 2013). In our review, both studies included only amnestic participants, therefore, we could predict a worse accuracy if non-amnestic $\mathrm{MCl}$ were included. Additionally, some other risk factors like family history of dementia, APOE $\epsilon 4$ allele presence, and $A \beta$ and tau protein levels in cerebrospinal fluid may contribute to a faster progression rate to dementia. In conclusion, further reviews that include high quality research with more detailed data about the characteristics of $\mathrm{MCl}$ are required to not only explore the underlying mechanisms but also to elucidate the causal pathways that link $18 \mathrm{~F}$ flutemetamol PET scan positivity of diverse $\mathrm{MCl}$ subtypes and disease progression.

\section{Strengths and weaknesses of the review}

We conducted an extensive, comprehensive, and sensitive literature search, using eleven different electronic databases without any limit to language or date. However, we were only able to include two studies with 243 participants, therefore, our DTA estimates are relatively imprecise. This paucity of evidence reflects the very significant challenges inherent in conducting long term prospective studies of well-characterised participants, followed up to the point of progression to clinical dementia. The methodological quality assessment and data syntheses were based on the recommended methods (Davis 2013). To increase the reliability of our findings, we included only studies that fulfilled delayed verification of progression from $\mathrm{MCl}$ to $\mathrm{ADD}$ or other form of dementia (non-ADD) or any form of dementia at follow up.

The included studies had significant methodological limitations that weakened confidence in the results of this SR. First, considerable uncertainty remains concerning the clinical diagnosis of ADD; the anatomopathological diagnosis would be the better way to probe the diagnosis, but there was not a clear definition of a positive index test in one study, the reference standard in one study was not explicitly described, and the major problem was the potential conflict of interest with the company that produced the tracer in both studies.

The selection of participants with $\mathrm{MCl}$ in these studies could be another weakness, because we did not have all the necessary baseline data in the ClinicalTrials.gov registered study included in this SR (NCT01028053), and what would happen in those with non-amnestic $\mathrm{MCl}$ in the future. However, this selection of participants, such as type of $\mathrm{MCl}$, age, presence of the APOE $\epsilon 4$ allele, structural abnormality at MRI, hypometabolism at FDG-PET scan, and alteration in cerebrospinal fluid could help determine different subgroups of people at higher risk of developing dementia at follow-up, and perform a stratification that could help avoid biases, and develop more efficient studies in the future (Caroli 2015; Hampel 2012; Wolz 2016). NCT01028053 had some information about age, presence of the APOE 64 allele, and amnestic $\mathrm{MCl}$ stage (early/late) in a Cox regression, but without useable data for this DTA review. The Thurfjell study tried to correlate SUVR and hippocampus volume (Thurfjell 2012).

Finally, an important weakness of this SR was the nonresponse of the authors about their studies. This has resulted in a lack of data for analysis in this review.

\section{Applicability of findings to the review question}

Regarding the question of this review: Could the 18 F-flutemetamol PET scan identify those $\mathrm{MCl}$ participants who would progress to clinical dementia at follow up?. There were applicability concerns in the index test in one study that did not provide a clear definition of what was considered a $18 \mathrm{~F}$-flutemetamol positive result. There were also applicability concerns in the reference standard in one study, mainly due to lack of information regarding how the clinical progression to ADD was made. However, due to the limited number of included studies and levels of heterogeneity with respect to the two domains mentioned above, it was difficult to determine to what extent the findings from this review could be applied to clinical practice. 
The DTA of the 18F-flutemetamol PET scan for identifying Alzheimer's disease pathology and identifying those people with $\mathrm{MCl}$ who would convert to ADD could be affected by a number of factors that have not been determined so far. First, and most important, is the lack of a large study to evaluate this question. We included only two studies that addressed the question with 243 participants at follow-up. Second, the quantitative criterion used for several studies is not the actually approved criterion of FDA and EMA, because they approved the visual assessment interpretation. However, in this SR we included one study with quantitative evaluation and the other with visual assessment, with lack of information regarding how that visual assessment was made.

We await new studies using the FDA and EMA approval visual assessment criteria in longitudinal studies. The $18 \mathrm{~F}$-flutemetamol test is expensive, therefore, we believe it is important to clearly determine its DTA prior to recommending its adoption in clinical practice. The actual sensitivity and specificity are too low to have enough accuracy to be used in clinical practice to predict the progression from $\mathrm{MCl}$ to $\mathrm{ADD}$.

\section{AUTHORS' CONCLUSIONS}

\section{Implications for practice}

As of today, the use of $18 \mathrm{~F}$-flutemetamol has not been established for predicting development of Alzheimer's disease (FDA 2014; EMA 2014), and is not indicated in people with $\mathrm{MCl}$, except in clinical trials and research studies ( Albert 2011).

However, the Amyloid Imaging Task Force, the Society of Nuclear Medicine and Molecular Imaging, and the Alzheimer's Association have proposed the usage of amyloid PET in people with persistent or progressive unexplained $\mathrm{MCl}$ (Johnson 2013). The DTA of 18 F-flutemetamol PET scans, as determined in this SR, has a variable sensitivity and specificity based on two studies with 243 participants at follow-up to predict the progression from $\mathrm{MCl}$ to ADD.

Due to the aforementioned and the methodological limitations of the included studies, it is not possible to recommend the routine use of $18 \mathrm{~F}$-flutemetamol in clinical practice. The $18 \mathrm{~F}$ flutemetamol biomarker is expensive, therefore it is important to clearly determine its DTA and to standardise the process for the diagnostic modality prior to it being recommended for clinical practice.

\section{Implications for research}

FDA and EMA have established the $18 \mathrm{~F}$-flutemetamol criteria positivity in order to use these in ADD patient evaluation and their use in $\mathrm{MCl}$ participants is accepted in research settings and clinical trials (Albert 2011). However, their use has also been proposed in clinical practice to evaluate people with $\mathrm{MCl}$ by the Nuclear Medicine Society and the Alzheimer's Association (Johnson 2013). It is still used in many studies with different $18 \mathrm{~F}$ flutemetamol SUVRs, visual assessment, or both. This promotes different accuracies for the tracer even in people with ADD when are compared with HC. Therefore, it is necessary to consider visual assessment as the most important option to interpret the $18 \mathrm{~F}$-flutemetamol PET scan, because this is the approach to the interpretation established by FDA and EMA (EMA 2014; FDA 2014).

On the other hand, clinical assessment in people with memory complaints is not always made with only one test; one could add different tests such as volumetric hyppocampal MRI, FDGPET, SPECT, CSF, and others. This suggestion has face validity because neurodegenerative diseases are complex disorders with occasionally multiple and overlapping pathophysiological processes. Multitracer imaging may be helpful in combining metabolic, inflammation, or apoptosis markers with those labelling typical protein aggregations seen in the progression of $\mathrm{MCl}$ to Alzheimer's disease. In future, various PET imaging modalities are needed to evaluate the usefulness of the various PET tracers as predictors of progression to Alzheimer's disease in $\mathrm{MCl}$ studies with clinical follow-up. There is a hypothesis that amyloid deposition is an early event in Alzheimer's disease that reaches a relative plateau even at the $\mathrm{MCl}$ stage, while downstream biomarkers measure neuronal loss and dysfunction, and cognitive measures are more dynamic at the symptomatic disease stage (Jack 2010). Based on this hypothesis, the combination of structural imaging, functional imaging, and cognitive tests may be better predictors of when an individual will convert. However, there is a lack of studies with ${ }^{18} \mathrm{~F}$ flutemetamol combined with other tests to predict the progression from $\mathrm{MCl}$ to $\mathrm{ADD}$ or any form of dementia.

Additionally, if we consider the hierarchical evidence needed for level of efficacy of diagnostic imaging tests, we are currently in the second step of six according to Herscovitch (Herscovitch 2015): technical efficacy, diagnostic accuracy efficacy, diagnostic thinking efficacy, therapeutic impact, patient health outcomes, and finally societal efficacy. Therefore, we need further research about accuracy before progressing to the other steps with their specific studies before we can incorporate the $18 \mathrm{~F}$-flutemetamol PET scan into clinical practice.

\section{ACKNOWLEDGEMENTS}

Gabriel Martínez is a PhD candidate in Methodology of Biomedical Research and Public Health at the Department of Paediatrics, Obstetrics and Gynaecology and Preventive Medicine, Universitat Autònoma de Barcelona, Barcelona, Spain.

We are grateful to the authors of included and excluded studies who responded to our requests for additional information.

We thank the Cochrane Dementia and Cognitive Improvement Group (CDCIG) for strong support, especially Sue Marcus in finalizing the review.

We thank Anna Noel-Storr, Information Specialist of the CDCIG, for her assistance with the design of the search strategy.

We thank Gerard Urrútia and Marta Roqué i Figuls for their contribution in the preparation of the protocol for the review (Martínez 2016)

We thank the peer reviewers for their many helpful suggestions. 


\section{R E F E R E N C E S}

\section{References to studies included in this review}

\section{NCT01028053 \{unpublished data only\}}

EUCTR2009-010227-62-GB. A principal open-label study to assess the prognostic usefulness of flutemetamol (F18) injection for identifying subjects with amnestic mild cognitive impairment who will convert to probable Alzheimer's disease. apps.who.int/trialsearch/Trial2.aspx? TrialID=EUCTR2009-010227-62-GB (first received 6 July 2009).

* NCT01028053. Assess the prognostic usefulness of flutemetamol (18F) injection for identifying subjects with amnestic mild cognitive impairment who will convert to clinically probable Alzheimer's disease. www.clinicaltrials.gov/ show/NCT01028053 (first received 9 December 2009).

\section{Thurfjell 2012 \{published data only\}}

Thurfjell L, Lötjönen J, Lundqvist R, Koikkalainen J, Soininen $\mathrm{H}$, Waldemar G, et al. Combination of biomarkers: PET [18F] flutemetamol imaging and structural MRI in dementia and mild cognitive impairment. Neuro-degenerative Diseases 2012;10:246-9

\section{References to studies excluded from this review}

\section{Goukasian 2015 \{published data only\}}

Goukasian N, LeClair H, Porat S, Hwang KS, Ringman JM, Silverman $\mathrm{D}$, et al. Anxiety is associated with brain amyloidosis in cognitively normal and mild cognitive impairment subjects: a [18f] flutemetamol PET study. Alzheimer's \& Dementia 2015;11(7 Suppl):17.

\section{Rowe 2015a \{published data only\}}

Rowe CC, Dore V, Bourgeat P, Brown BM, Thurfjell L, Macaulay L, et al. Abeta accumulation in non-demented individuals: a longitudinal F-18-flutemetamol study. Alzheimer's \& Dementia. 2015; Vol. 11:125.

\section{Rowe 2015b \{published data only\}}

Rowe CC, Dore V, Bourgeat P, Thurfjell L, Macaulay L, Williams R, et al. Longitudinal assessment of abeta accumulation in nondemented individuals: a 18F-flutemetamol study. Neurodegenerative Diseases 2015;15 Suppl 1:904.

Rowe 2015c \{published data only\}

Rowe C, Dore V, Bourgeat P, Thurfjell L, Macaulay S, Williams R, et al. Longitudinal assessment of Abeta accumulation in nondemented individuals: a 18F-flutemetamol study. Journal of Nuclear Medicine 2015;56:193.

\section{References to ongoing studies}

\section{EUCTR2011-001756-12-BE \{unpublished data only\}}

EUCTR2011-001756-12-BE. Surrogate markers evaluation in pre-demented Alzheimer's disease patients and healthy elderly controls. apps.who.int/trialsearch/Trial2.aspx? TrialID=EUCTR2011-001756-12-BE (first received 11 April 2012).
EUCTR2011-006195-39-SE \{unpublished data only\}

EUCTR2011-006195-39-SE. An open-label study to compare the prognostic value of (18F)Flutemetamol PET-imaging with longitudinal biomarker data in healthy volunteers and patients with mild cognitive impairment. apps.who.int/trialsearch/ Trial2.aspx?TrialID=EUCTR2011-006195-39-SE (first received 17 January 2012).

EUCTR2016-002635-15-NL \{unpublished data only\}

EUCTR2016-002635-15-NL. Study to Identify Factors associated with Resilience to Clinical Dementia at Old Age - 90+ Study. apps.who.int/trialsearch/Trial2.aspx? TrialID=EUCTR2016-002635-15-NL (first received 14 July 2016).

EUCTR2017-000094-36-SE \{unpublished data only\}

EUCTR2017-000094-36-SE. The BioFINDER 2 study improved diagnostics and increased understanding of the pathophysiology of cognitive disorders. apps.who.int/ trialsearch/Trial2.aspx?TrialID=EUCTR2017-000094-36-SE (first received 30 January 2017).

\section{JPRN-UMIN000019926 \{unpublished data only\}}

JPRN-UMIN000019926. Clinical and neuroimaging study on preclinical Alzheimer's disease. apps.who.int/trialsearch/ Trial2.aspx?TrialID=JPRN-UMIN000019926 (first received 1 December 2015)

NCT02164643 \{unpublished data only\}

NCT02164643. Longitudinal study of brain amyloid imaging in MEMENTO (MEMENTOAmyGing). www.clinicaltrials.gov/show/ NCT02164643 (first received16 June 2014).

NCT02196116 \{unpublished data only\}

NCT02196116. Amyloïd load in elderly population: effect of cognitive reserve (EDUMA). www.clinicaltrials.gov/show/ NCT02196116 (first received 21 July 2014).

\section{Additional references}

\section{Albert 2011}

Albert MS, DeKosky ST, Dickson D, Dubois B, Feldman HH, Fox NC, et al. The diagnosis of mild cognitive impairment due to Alzheimer's disease: recommendations from the National Institute on Aging-Alzheimer's Association workgroups on diagnostic guidelines for Alzheimer's disease. Alzheimer's \& Dementia: the Journal of the Alzheimer's Association 2011;7(3):270-9.

\section{Alzheimer's Association 2010}

Alzheimer's Association. Changing the Trajectory of Alzheimer's Disease: A National Imperative. Alzheimer's Association 2010.

\section{APA 1987}

American Psychiatric Association. Diagnostic and Statistical Manual of Mental Disorders. 3rd Edition. Washington, DC: American Psychiatric Association, 1987. 


\section{APA 1994}

American Psychiatric Association. Diagnostic and Statistical Manual of Mental Disorders. 4th Edition. Washington, DC: American Psychiatric Association, 1994.

\section{Archer 2015}

Archer HA, Smailagic N, John C, Holmes RB, Takwoingi Y, Coulthard EJ, et al. Regional Cerebral Blood Flow Single Photon Emission Computed Tomography for detection of Frontotemporal dementia in people with suspected dementia. Cochrane Database of Systematic Reviews 2015, Issue 6. [DOI: 10.1002/14651858.CD010896.pub2]

\section{Arevalo-Rodriguez 2015}

Arevalo-Rodriguez I, Smailagic N, Roqué i Figuls M, Ciapponi A, Sanchez-Perez E, Giannakou A, et al. Mini-Mental State Examination (MMSE) for the detection of Alzheimer's disease and other dementias in people with mild cognitive impairment (MCI). Cochrane Database of Systematic Reviews 2015, Issue 3. [DOI: 10.1002/14651858.CD010783.pub2]

\section{Bossuyt 2008}

Bossuyt PM, Leeflang MM. Chapter 6: Developing criteria for including studies. In: Cochrane Handbook for Systematic Reviews of Diagnostic Test Accuracy Version 0.4 [updated September 2008]. The Cochrane Collaboration, 2008. http://methods.cochrane.org/sdt/sites/ methods.cochrane.org.sdt/files/uploads/Chapter06-IncludingStudies\%20(September-2008).pdf. The Cochrane Collaboration.

\section{Boxer 2005}

Boxer AL, Miller BL. Clinical features of frontotemporal dementia. Alzheimer Disease and Associated Disorders 2005;19(Suppl 1):S3-6.

\section{Brun 1994}

Brun A, Englund B, Gustafson L, Passant U, Mann DMA, Neary D, et al. Clinical and neuropathological criteria for frontotemporal dementia. The Lund and Manchester Groups. Journal of Neurology, Neurosurgery, and Psychiatry 1994;57(4):416-8.

\section{Bruscoli 2004}

Bruscoli M, Lovestone S. Is $\mathrm{MCI}$ really just early dementia? A systematic review of conversion studies. International Psychogeriatrics/IPA 2004;16(2):129-40.

\section{Caroli 2015}

Caroli A, Prestia A, Wade S, Chen K, Ayutyanont N, Landau SM, et al. Alzheimer Disease Biomarkers as Outcome Measures for Clinical Trials in $\mathrm{MCl}$. Alzheimer disease and associated disorders 2015;29(2):101-9.

\section{Chan 2014}

Chan CCH, Fage BA, Smailagic N, Gill SS, Herrmann N, Nikolaou V, et al. Mini-Cog for the diagnosis of Alzheimer's disease dementia and other dementias within a secondary care setting. Cochrane Database of Systematic Reviews 2014, Issue 12. [DOI: 10.1002/14651858.CD011414]

\section{CMS 2013}

The Centers for Medicare \& Medicaid Services. Decision Memo for Beta Amyloid Positron Emission Tomography in Dementia and Neurodegenerative Disease (CAG-00431N). https://www.cms.gov/medicare-coverage-database/details/ nca-decision-memo.aspx?NCAld=265 (accessed 08/10/2015).

\section{Cordella 2013}

Cordella CB, Borson S, Boustani M, Chodosh J, Reuben D, Verghese J, et al. Alzheimer's Association recommendations for operationalizing the detection of cognitive impairment during the Medicare Annual Wellness Visit in a primary care setting. Alzheimer's \& Dementia: the Journal of the Alzheimer's Association 2013;9(2):141-50.

\section{Creavin 2016}

Creavin S, Wisniewski S, Noel-Storr A, Trevelyan C, Hampton T, Rayment D, et al. Mini-Mental State Examination (MMSE) for the detection of dementia in clinically unevaluated people aged 65 and over in community and primary care populations. Cochrane Database of Systematic Reviews 2016, Issue 4. [DOI: 10.1002/14651858.CD011145.pub2]

\section{Davis 2013}

Davis DHJ, Creavin ST, Noel-Storr A, Quinn TJ, Smailagic N, Hyde $C$, et al. Neuropsychological tests for the diagnosis of Alzheimer's disease dementia and other dementias: a generic protocol for cross-sectional and delayed-verification studies. Cochrane Database of Systematic Reviews 2013, Issue 3. [DOI: 10.1002/14651858.CD010460]

\section{Davis 2015}

Davis DHJ, Creavin ST, Yip JLY, Noel-Storr AH, Brayne C, Cullum S. Montreal Cognitive Assessment for the diagnosis of Alzheimer's disease and other dementias. Cochrane Database of Systematic Reviews 2015, Issue 10. [DOI: 10.1002/14651858.CD010775.pub2]

\section{De la Torre 2004}

de la Torre JC. Is Alzheimer's disease a neurodegenerative or a vascular disorder? Data, dogma, and dialectics. The Lancet. Neurology 2004;3(3):184-90.

\section{Dubois 2014}

Dubois B, Feldman HH, Jacova C, Hampel H, Molinuevo JL, Blennow K, et al. Advancing research diagnostic criteria for Alzheimer's disease: the IWG-2 criteria. The Lancet. Neurology 2014;13(6):614-29.

\section{Elias-Sonnenschein 2014a}

Elias-Sonnenschein LS, Viechtbauer W, Ramakers I, Ukoumunne O, Verhey FRJ, Visser PJ. APOE- $€ 4$ allele for the diagnosis of Alzheimer's and other dementia disorders in people with mild cognitive impairment in a community setting. Cochrane Database of Systematic Reviews 2014, Issue 1. [DOI: 10.1002/14651858.CD010948]

\section{Elias-Sonnenschein 2014b}

Elias-Sonnenschein LS, Viechtbauer W, Ramakers I, Ukoumunne O, Verhey FRJ, Visser PJ. APOE- $€ 4$ allele for the diagnosis of Alzheimer's and other dementia disorders in

$18 \mathrm{~F}$ PET with flutemetamol for the early diagnosis of Alzheimer's disease dementia and other dementias in people with mild cognitive impairment (MCI) (Review)

Copyright $\odot 2017$ The Cochrane Collaboration. Published by John Wiley \& Sons, Ltd. 
people with mild cognitive impairment in a primary care setting. Cochrane Database of Systematic Reviews 2014, Issue 1. [DOI: 10.1002/14651858.CD010949]

\section{Elias-Sonnenschein 2014c}

Elias-Sonnenschein LS, Viechtbauer W, Ramakers I, Ukoumunne O, Verhey FRJ, Visser PJ. APOE- $€ 4$ allele for the diagnosis of Alzheimer's and other dementia disorders in people with mild cognitive impairment in a secondary care setting. Cochrane Database of Systematic Reviews 2014, Issue 1. [DOI: 10.1002/14651858.CD010950]

\section{EMA 2014}

European Medicines Agency. Vizamyl. Annex 1. Summary of product characteristics. www.ema.europa.eu/docs/en_ GB/document_library/EPAR_-_Product_Information/ human/002557/WC500172950.pdf (accessed 8 April 2015).

\section{Espinosa 2013}

Espinosa A, Alegret M, Valero S, Vinyes-Junque G, Hernandez I, Mauleon A, et al. A longitudinal follow-up of 550 mild cognitive impairment patients: evidence for large conversion to dementia rates and detection of major risk factors involved. Journal of Alzheimer's Disease 2013;34(3):769-80.

\section{Fage 2015}

Fage BA, Chan CCH, Gill SS, Noel-Storr AH, Herrmann N, Smailagic N, et al. Mini-Cog for the diagnosis of Alzheimer's disease dementia and other dementias within a community setting. Cochrane Database of Systematic Reviews 2015, Issue 2. [DOI: 10.1002/14651858.CD010860.pub2]

\section{FDA 2014}

Food, Drug Administration. Vizamyl. www.accessdata.fda.gov/ drugsatfda_docs/label/2014/203137s002lbl.pdf (accessed 8 April 2015).

\section{Filippini 2012}

Filippini G, Casazza G, Bellatorre AG, Lista C, Duca P, Beecher D, et al. The role of MRI in the early diagnosis of Alzheimer's disease or other dementias in persons with mild cognitive impairment (MCl). Cochrane Database of Systematic Reviews 2012, Issue 2. [DOI: 10.1002/14651858.CD009628]

\section{Garcia-Alloza 2011}

Garcia-Alloza M, Gregory J, Kuchibhotla KV, Fine S, Wei Y, Ayata $\mathrm{C}$, et al. Cerebrovascular lesions induce transient $\beta$-amyloid deposition. Brain: a Journal of Neurology 2011;134(12):3697-707.

\section{Gelber 2012}

Gelber RP, Launer LJ, White LR. The Honolulu-Asia Aging Study: epidemiologic and neuropathologic research on cognitive impairment. Current Alzheimer research 2012;9(6):664-72.

\section{Geslani 2005}

Geslani DM, Tierney MC, Herrmann N, Szalai JP. Mild cognitive impairment: an operational definition and its conversion rate to Alzheimer's disease. Dementia and Geriatric Cognitive Disorders 2005;19(5-6):383-9.

\section{Goedert 2006}

Goedert M, Spillantini MG. A century of Alzheimer's disease. Science 2006;314(5800):777-81.

\section{Hampel 2012}

Hampel H, Lista S, Khachaturian Z. Development of biomarkers to chart all Alzheimer's disease stages: The royal road to cutting the therapeutic Gordian Knot. Alzheimer's \& dementia : the journal of the Alzheimer's Association 2012;8(4):312-36.

\section{Harrison 2014}

Harrison JK, Fearon P, Noel-Storr AH, McShane R, Stott DJ, Quinn TJ. Informant Questionnaire on Cognitive Decline in the Elderly (IQCODE) for the diagnosis of dementia within a general practice (primary care) setting. Cochrane Database of Systematic Reviews 2014, Issue 7. [DOI: 10.1002/14651858.CD010771.pub2]

\section{Harrison 2015}

Harrison JK, Fearon P, Noel-Storr AH, McShane R, Stott DJ, Quinn TJ. Informant Questionnaire on Cognitive Decline in the Elderly (IQCODE) for the diagnosis of dementia within a secondary care setting. Cochrane Database of Systematic Reviews 2015, Issue 3. [DOI: 10.1002/14651858.CD010772.pub2]

\section{Hendry 2014}

Hendry K, Lees RA, McShane R, Noel-Storr AH, Stott DJ, Quinn TJ. AD-8 for diagnosis of dementia across a variety of healthcare settings. Cochrane Database of Systematic Reviews 2014, Issue 5. [DOI: 10.1002/14651858.CD011121]

\section{Herscovitch 2015}

Herscovitch P. Regulatory approval and insurance reimbursement: the final steps in clinical translation of amyloid brain imaging. Clinical and translational imaging 2015;3:75-7.

\section{Heyden 2013}

Hayden EY, Teplow DB. Amyloid $\beta$-protein oligomers and Alzheimer's disease. Alzheimer's research \& therapy 2013;5(6):1-11.

\section{Hyman 2012}

Hyman BT, Phelps CH, Beach TG, Bigio EH, Cairns NJ, Carrillo MC, et al. National Institute on Aging-Alzheimer's Association guidelines for the neuropathologic assessment of Alzheimer's disease. Alzheimer's \& dementia : the journal of the Alzheimer's Association 2012;8(1):1-13.

\section{ICD-10}

World Health Organization. International Statistical Classification of Diseases and Related Health Problems (ICD-10 Version:2010). http://apps.who.int/classifications/icd10/ browse/2010/en (accessed 29 January 2015).

\section{Jack 2010}

Jack CR Jr, Knopman DS, Jagust WJ, Shaw LM, Aisen PS, Weiner MW. Hypothetical model of dynamic biomarkers of the Alzheimer's pathological cascade. Lancet Neurology 2010;9(1):119-28 


\section{Jellinger 2006}

Jellinger K. Clinicopathological analysis of dementia disorders in the elderly--an update. Journal of Alzheimer's Disease: JAD 2006;9(Supplement 3):61-70.

\section{Johnson 2013}

Johnson KA, Minoshima S, Bohnen NI, Donohoe KJ, Foster NL, Herscovitch P, et al. Appropriate use criteria for amyloid PET: a report of the Amyloid Imaging Task Force, the Society of Nuclear Medicine and Molecular Imaging, and the Alzheimer's Association. Journal of nuclear medicine : official publication, Society of Nuclear Medicine 2013;54(3):476-90.

\section{Knottnerus 2002}

Knottnerus JA, van Weel C, Muris JW. Evaluation of diagnostic procedures. BMJ 2002;324(7335):477-80.

\section{Kobylecki 2015}

Kobylecki C, Langheinrich T, Hinz R, Vardy ER, Brown G, Martino ME, et al. 18F-Florbetapir PET in patients with frontotemporal dementia and Alzheimer disease. Journal of Nuclear Medicine 2015;56(3):386-91.

\section{Kokkinou 2014}

Kokkinou M, Smailagic N, Noel-Storr AH, Hyde C, Ukoumunne O, Worrall RE, et al. Plasma and Cerebrospinal fluid (CSF) Abeta42 for the differential diagnosis of Alzheimer's disease dementia in participants diagnosed with any dementia subtype in a specialist care setting. Cochrane Database of Systematic Reviews 2014, Issue 1. [DOI: 10.1002/14651858.CD010945]

\section{Koole 2009}

Koole M, Lewis DM, Buckley C, Nelissen N, Vandenbulcke M, Brooks DJ, et al. Whole-body biodistribution and radiation dosimetry of 18F-GE067: a radioligand for in vivo brain amyloid imaging. Journal of Nuclear Medicine 2009;50(5):818-22.

\section{Lees 2014}

Lees RA, Stott DJ, McShane R, Noel-Storr AH, Quinn TJ. Informant Questionnaire on Cognitive Decline in the Elderly (IQCODE) for the early diagnosis of dementia across a variety of healthcare settings. Cochrane Database of Systematic Reviews 2014, Issue 10. [DOI: 10.1002/14651858.CD011333]

\section{Lundh 2017}

Lundh A, Lexchin J, Mintzes B, Schroll JB, Bero L. Industry sponsorship and research outcome. Cochrane Database of Systematic Reviews 2017, Issue 2. [DOI: 10.1002/14651858.MR000033.pub3]

\section{Ma 2014}

Ma Y, Zhang S, Li J, Zheng DM, Guo Y, Feng J, et al. Predictive accuracy of amyloid imaging for progression from mild cognitive impairment to Alzheimer disease with different lengths of follow-up: a meta-analysis. Medicine 2014;93(27):1-12.

\section{Martínez 2016}

Martínez G, Flicker L, Vernooij RWM, Fuentes Padilla P, Zamora J, Roqué i Figuls M, et al. ${ }^{18} \mathrm{~F}$ PET ligands for the early diagnosis of Alzheimer's disease dementia and other dementias in people with mild cognitive impairment $(\mathrm{MCl})$. Cochrane Database of Systematic Reviews 2016, Issue 5. [DOI: 10.1002/14651858.CD012216]

\section{Matthews 2008}

Matthews FE, Stephan BC, McKeith IG, Bond J, Brayne C, Medical Research Council Cognitive Function and Ageing Study. Two-year progression from mild cognitive impairment to dementia: to what extent do different definitions agree? Journal of the American Geriatrics Society 2008;56(8):1424-33.

\section{Mattsson 2009}

Mattsson N, Zetterberg H, Hansson O, Andreasen N, Parnetti L, Jonsson $\mathrm{M}$, et al. CSF biomarkers and incipient Alzheimer disease in patients with mild cognitive impairment. JAMA 2009;302(4):385-93.

\section{McCleery 2015}

McCleery J, Morgan S, Bradley KM, Noel-Storr AH, Ansorge O, Hyde C. Dopamine transporter imaging for the diagnosis of dementia with Lewy bodies. Cochrane Database of Systematic Reviews 2015, Issue 1. [DOI: 10.1002/14651858.CD010633.pub2]

\section{McKeith 1996}

McKeith IG, Galasko D, Kosaka K, Perry EK, Dickson DW, Hansen LA, et al. Consensus guidelines for the clinical and pathologic diagnosis of dementia with Lewy bodies (DLB): report of the consortium on DLB international workshop. Neurology 1996;47(5):1113-24.

\section{McKeith 2005}

McKeith IG, Dickson DW, Lowe J, Emre M, O'Brien JT, Feldman H, et al for the Consortium on DLB. Diagnosis and management of dementia with Lewy bodies: third report of the DLB consortium. Neurology 2005;65(12):1863-72.

\section{McKhann 1984}

McKhann GM, Drachman D, Folstein M, Katzman R, Price D, Stadlan EM. Clinical diagnosis of Alzheimer's disease: report of the NINCDS-ADRDA Work Group under the auspices of Department of Health and Human Services Task Force on Alzheimer's Disease. Neurology 1984;34(7):939-44.

\section{McKhann 2011}

McKhann GM, Knopman DS, Chertkow H, Hyman BT, Jack CR Jr, Kawas $\mathrm{CH}$, et al. The diagnosis of dementia due to Alzheimer's disease: recommendations from the National Institute on Aging-Alzheimer's Association workgroups on diagnostic guidelines for Alzheimer's disease. Alzheimer's \& Dementia: the Journal of the Alzheimer's Association 2011;7(3):263-9.

\section{Mitchell 2009}

Mitchell AJ, Shiri-Feshki M. Rate of progression of mild cognitive impairment to dementia--meta-analysis of 41 robust inception cohort studies. Acta Psychiatrica Scandinavica 2009;119(4):252-65.

\section{Morris 1993}

Morris JC. The Clinical Dementia Rating (CDR): current version and scoring rules. Neurology 1993;43(11):2412-4.

18F PET with flutemetamol for the early diagnosis of Alzheimer's disease dementia and other dementias in people with mild cognitive impairment ( $\mathrm{MCI}$ ) (Review)

Copyright (c) 2017 The Cochrane Collaboration. Published by John Wiley \& Sons, Ltd. 


\section{NAO 2007}

National Audit Office. Improving services and support for people with dementia. Report by the Comptroller and Auditor General. HC 604 Session General 2006-2007. 4 July 2007. https:// www.nao.org.uk/wp-content/uploads/2007/07/0607604.pdf (accessed 25th March 2015).

\section{Neary 1998}

Neary D, Snowden JS, Gustafson L, Passant U, Stuss D, Black S, et al. Frontotemporal lobar degeneration: a consensus on clinical diagnostic criteria. Neurology 1998;51(6):1546-54.

\section{Nelissen 2009}

Nelissen N, Van Laere K, Thurfjell L, Owenius R, Vandenbulcke $\mathrm{M}$, Koole $\mathrm{M}$, et al. Phase 1 study of the Pittsburgh compound $\mathrm{B}$ derivative $18 \mathrm{~F}$-flutemetamol in healthy volunteers and patients with probable Alzheimer disease. Journal of Nuclear Medicine 2009;50(8):1251-9.

\section{NICE 2006}

National Institute for Health Care Excellence. Dementia: supporting people with dementia and their carers in health and social care. NICE guidelines [CG42] Published date: November 2006. Last updated: April 2014. https://www.nice.org.uk/ guidance/cg42 (accessed 17th April 2015).

\section{Noel-Storr 2013}

Noel-Storr AH, Flicker L, Ritchie CW, Nguyen GH, Gupta T, Wood P, et al. Systematic review of the body of evidence for the use of biomarkers in the diagnosis of dementia. Alzheimer's \& Dementia: the Journal of the Alzheimer's Association 2013;9(3):e96-105.

\section{Noel-Storr 2014}

Noel-Storr AH, McCleery JM, Richard E, Ritchie CW, Flicker L, Cullum SJ, et al. Reporting standards for studies of diagnostic test accuracy in dementia: The STARDdem Initiative. Neurology 2014;83(4):364-73.

\section{Okello 2007}

Okello A, Edison P, Archer H, Hinz R, Fox N, Kennedy AM, et al. Amyloid deposition and cerebral glucose metabolism in mild cognitive impairment: a longitudinal 11C-PIB and 18F-FDG PET Study. Journal of Neurology, Neurosurgery, and Psychiatry 2007;78(2):219-20

\section{Petersen 1999}

Petersen RC, Smith GE, Waring SC, Ivnik RJ, Tangalos EG, Kokmen E. Mild cognitive impairment: clinical characterization and outcome. Archives of Neurology 1999;56(3):303-8.

\section{Petersen 2004}

Petersen RC. Mild cognitive impairment as a diagnostic entity. Journal of Internal Medicine 2004;256(3):183-94.

\section{Petersen 2009}

Petersen RC, Roberts RO, Knopman DS, Boeve BF, Geda YE, Ivnik RJ, et al. Mild cognitive impairment: ten years later. Archives of Neurology 2009;66(12):1447-55.

\section{Quinn 2014}

Quinn TJ, Fearon P, Noel-Storr AH, Young C, McShane R, Stott DJ. Informant Questionnaire on Cognitive Decline in the Elderly (IQCODE) for the diagnosis of dementia within community dwelling populations. Cochrane Database of Systematic Reviews 2014, Issue 4. [DOI: 10.1002/14651858.CD010079.pub2]

\section{Rascovsky 2011}

Rascovsky K, Hodges JR, Knopman D, Mendez MF, Kramer JH, Neuhaus J, et al. Sensitivity of revised diagnostic criteria for the behavioural variant of frontotemporal dementia. Brain: $a$ Journal of Neurology 2011;134(Pt 9):2456-77.

\section{Review Manager 2014 [Computer program]}

The Nordic Cochrane Centre, The Cochrane Collaboration. Review Manager. Version 5.3. Copenhagen: The Nordic Cochrane Centre, The Cochrane Collaboration, 2014.

\section{Richard 2012}

Richard E, Schmand B, Eikelenboom P, Westendorp RG, Van Gool WA. The Alzheimer myth and biomarker research in dementia. Journal of Alzheimer's Disease: JAD 2012;31(Suppl 3):S203-9.

\section{Ritchie 2013}

Ritchie C, Smailagic N, Ladds EC, Noel-Storr AH, Ukoumunne O, Martin S. CSF tau and the CSF tau/ABeta ratio for the diagnosis of Alzheimer's disease dementia and other dementias in people with mild cognitive impairment $(\mathrm{MCl})$. Cochrane Database of Systematic Reviews 2013, Issue 10. [DOI: 10.1002/14651858.CD010803]

\section{Ritchie 2014}

Ritchie C, Smailagic N, Noel-Storr AH, Takwoingi Y, Flicker L, Mason SE, et al. Plasma and cerebrospinal fluid amyloid beta for the diagnosis of Alzheimer's disease dementia and other dementias in people with mild cognitive impairment $(\mathrm{MCl})$. Cochrane Database of Systematic Reviews 2014, Issue 6. [DOI: 10.1002/14651858.CD008782.pub4]

\section{Román 1993}

Román GC, Tatemichi TK, Erkinjuntti T, Cummings JL, Masdeu JC, Garcia JH, et al. Vascular dementia: diagnostic criteria for research studies. Report of the NINDS-AIREN International Workshop. Neurology 1993;43(2):250-60.

\section{Royall 2014}

Royall DR, Palmer RF. The temporospatial evolution of neuritic plaque-related and independent tauopathies: implications for dementia staging. Journal of Alzheimer's Disease 2014;40(3):541-9.

\section{Savva 2009}

Savva GM, Wharton SB, Ince PG, Forster G, Matthews FE, Brayne C, Medical Research Council Cognitive Function and Ageing Study. Age, neuropathology, and dementia. The New England Journal of Medicine 2009;360(22):2302-9. 


\section{Schneider 2007}

Schneider JA, Arvanitakis Z, Bang W, Bennett DA. Mixed brain pathologies account for most dementia cases in communitydwelling older persons. Neurology 2007;69(24):2197-204.

\section{Schneider 2009}

Schneider JA, Arvanitakis Z, Leurgans SE, Bennett DA. The neuropathology of probable Alzheimer disease and mild cognitive impairment. Annals of neurology 2009;66(2):200-8.

\section{Seitz 2014}

Seitz DP, Fage BA, Chan CCH, Gill SS, Herrmann N, Smailagic N, et al. Mini-Cog for the diagnosis of Alzheimer's disease dementia and other dementias within a primary care setting. Cochrane Database of Systematic Reviews 2014, Issue 12. [DOI: 10.1002/14651858.CD011415]

\section{Selkoe 2016}

Selkoe DJ, Hardy J. The amyloid hypothesis of Alzheimer's disease at 25 years. EMBO molecular medicine 2016;8(6):595-608.

\section{Serrano-Pozo 2013}

Serrano-Pozo A, Qian J, Monsell SE, Frosch MP, Betensky RA, Hyman BT. Examination of the clinicopathologic continuum of Alzheimer disease in the autopsy cohort of the National Alzheimer Coordinating Center. Journal of Neuropathology and Experimental Neurology 2013;72(12):1182-92.

\section{Smailagic 2015}

Smailagic N, Vacante M, Hyde C, Martin S, Ukoumunne O, Sachpekidis C. 18F-FDG PET for the early diagnosis of Alzheimer's disease dementia and other dementias in people with mild cognitive impairment $(\mathrm{MCl})$. Cochrane Database of Systematic Reviews 2015, Issue 1. [DOI: 10.1002/14651858.CD010632.pub2]

\section{Sperling 2011}

Sperling RA, Aisen PS, Beckett LA, Bennett DA, Craft S, Fagan AM, et al. Toward defining the preclinical stages of Alzheimer's disease: recommendations from the National Institute on Aging-Alzheimer's Association workgroups on diagnostic guidelines for Alzheimer's disease. Alzheimer's \& Dementia: the Journal of the Alzheimer's Association 2011;7(3):280-92.

\section{Thal 2015}

Thal DR, Beach TG, Zanette M, Heurling K, Chakrabarty A, Ismail A, et al. [(18)F]flutemetamol amyloid positron emission tomography in preclinical and symptomatic Alzheimer's disease: specific detection of advanced phases of amyloid- $\beta$ pathology. Alzheimer's \& Dementia 2015;11(8):975-85.

\section{Vandenberghe 2010}

Vandenberghe R, Van Laere K, Ivanoiu A, Salmon E, Bastin C, Triau E, et al. 18F-flutemetamol amyloid imaging in Alzheimer

\section{CHARACTERISTICS OF STUDIES}

Characteristics of included studies [ordered by study ID] disease and mild cognitive impairment: a phase 2 trial. Annals of Neurology 2010;68(3):319-29.

\section{Villemagne 2011}

Villemagne VL, Ong K, Mulligan RS, Holl G, Pejoska S, Jones G, et al. Amyloid imaging with (18)F-florbetaben in Alzheimer disease and other dementias. Journal of Nuclear Medicine 2011;52(8):1210-7.

\section{Visser 2006}

Visser PJ, Kester A, Jolles J, Verhey F. Ten-year risk of dementia in subjects with mild cognitive impairment. Neurology 2006;67(7):1201-7.

\section{White 2009}

White L. Brain lesions at autopsy in older Japanese-American men as related to cognitive impairment and dementia in the final years of life: a summary report from the HonoluluAsia aging study. Journal of Alzheimer's Disease: JAD 2009;18(3):713-25.

\section{Whiting 2011}

Whiting PF, Rutjes AWS, Westwood ME, Mallet S, Deeks JJ, Reitsma JB. QUADAS-2: a revised tool for the quality assessment of diagnostic accuracy studies. Annals of Internal Medicine 2011;155(8):529-36.

\section{WHO 2012}

World Health Organization, Alzheimer's Disease International. Dementia: a public health priority. 2012. http://www.who.int/ mental_health/publications/dementia_report_2012/en/.World Health Organization, (accessed 23th September 2015).

\section{Winblad 2004}

Winblad B, Palmer K, Kivipelto M, Jelic V, Fratiglioni L, Wahlund LO, et al. Mild cognitive impairment--beyond controversies, towards a consensus: report of the International Working Group on Mild Cognitive Impairment. Journal of Internal Medicine 2004;256(3):240-6.

\section{Wolz 2016}

Wolz R, Schwarz AJ, Gray KR, Yu P, Hill DL, Alzheimer's Disease Neuroimaging Initiative. Enrichment of clinical trials in $\mathrm{MCl}$ due to $A D$ using markers of amyloid and neurodegeneration. Neurology 2016;87(12):1235-41.

\section{Zhang 2014}

Zhang S, Smailagic N, Hyde C, Noel-Storr AH, Takwoingi Y, McShane R, et al. 11C-PIB-PET for the early diagnosis of Alzheimer's disease dementia and other dementias in people with mild cognitive impairment $(\mathrm{MCl})$. Cochrane Database of Systematic Reviews 2014, Issue 7. [DOI: 10.1002/14651858.CD010386.pub2]

* Indicates the major publication for the study 
NCT01028053

\section{Study characteristics}

Patient sampling - There were 230 amnestic $\mathrm{MCl}$ participants that were evaluable for efficacy.

- The participants were 60 years old or older (US inclusion criteria in clinicaltrials.gov) or over 55 years old (Europe in EUDRACT).

- All participants met the Petersen criteria for amnestic $\mathrm{MCl}$ (details of the criteria not provided), had a score of less than or equal to 4 on the Modified Hachinski Ischemic Scale, a MMSE score of 24 to 30, and non-contrast MRI examination as part of the screening visit that excluded amnestic $\mathrm{MCl}$ arising from structural causes, and had not any significant neurologic disease other than suspected amnestic $\mathrm{MCl}$.

- No further details of participant sampling and recruitment were reported.

Patient characteristics and setting
- 232 amnestic $\mathrm{MCl}$ participants diagnosed by Petersen criteria (not reported which one of Petersen criteria was used)

- Gender: 114 male, 118 female

- Mean \pm SD age:71.1 \pm 8.62 years, 63 participants were less than 65 years old

- APOE $\epsilon 4$ carrier: not reported

- MMSE: not reported

- Years of education: not reported

- Sources of referral: not reported

- Setting: not reported
Index tests

- No data were given regarding the PET/CT scanner used in the different centres. Each participant received one $185 \mathrm{MBq}$ intravenous dose of $18 \mathrm{~F}$-flutemetamol Injection ( $\leq 10 \mathrm{mcg}$ total flutemetamol) injected within 40 seconds. A $185 \mathrm{MBq}$ dose exposes the subject to an effective dose of $5.92 \mathrm{mSv}$ of radiation.

- PET imaging started approximately 90 minutes after dosing. Imaging data were collected for 30 minutes in six 5-minute frames. Images were assessed visually by 5 blinded, independent, and trained readers. Based on the blinded image evaluation, each of 5 independent readers separately categorized each subject as having either 'normal' (negative for $A \beta$ ) or 'abnormal' (positive for $A \beta$ uptake) based on the PET image pattern.

- No further details were given regarding the index test.
Target condition and reference standard(s)
- Target condition: Alzheimer's disease dementia

- Reference standard: NINCDS-ADRDA criteria for ADD (McKhann 1984), and a CAC (consisted of 4 experts in the diagnosis of memory disorders) determined if the participant progressed or not to probable ADD, blinded to the investigator's progression assessment, flutemetamol and any other amyloid imaging data.

\section{Flow and timing}

- Duration of follow-up: 3 years

- Number included in analysis: 224 participants: 97 18 F-flutemetamol (+) and 127 18F-flutemetamol (-)

- Progression from $\mathrm{MCl}$ to ADD:

- 97 18F-flutemetamol (+): $52 \mathrm{MCl}$-converted to ADD and $45 \mathrm{MCl}$-not converted to ADD; 127 $18 \mathrm{~F}$-flutemetamol (-): $29 \mathrm{MCl}$-converted to ADD and $98 \mathrm{MCl}$-not converted to ADD

- $\mathrm{TP}=52 ; \mathrm{FP}=45 ; \mathrm{FN}=29 ; \mathrm{TN}=98$

- 8 participants withdrew prior to the first Clinical Adjudication Committee (CAC) evaluation

- Full financial support from the manufacturer of 18 F-flutemetamol tracer

\section{Methodological quality}

18F PET with flutemetamol for the early diagnosis of Alzheimer's disease dementia and other dementias in people with mild cognitive impairment (MCI) (Review)

Copyright $\odot 2017$ The Cochrane Collaboration. Published by John Wiley \& Sons, Ltd. 
NCT01028053 (Continued)

$\begin{array}{llll}\text { Item } & \text { Authors' judgement } & \text { Risk of bias } & \text { Applicability concerns }\end{array}$

\section{DOMAIN 1: Patient Selection}

Was a consecutive or random Unclear sample of patients enrolled?

Was a case-control design avoid- Yes ed?

\section{Did the study avoid inappropriate Unclear} exclusions?

\begin{tabular}{lll}
\hline & Unclear & Low \\
\hline DOMAIN 2: Index Test All tests &
\end{tabular}

\section{DOMAIN 2: Index Test All tests}

\section{Were the index test results inter- Yes} preted without knowledge of the results of the reference standard?

If a threshold was used, was it Unclear pre-specified?

\section{Was the 18F-flutemetamol PET Yes} scan interpretation done by a trained reader physician?

Did the study provide a clear defi- Unclear nition of what was considered to be a $18 \mathrm{~F}$-flutemetamol positive result?

\begin{tabular}{lll}
\hline & Unclear & Unclear \\
\hline
\end{tabular}

\section{DOMAIN 3: Reference Standard}

Is the reference standards like- Yes ly to correctly classify the target condition?

Were the reference standard results interpreted without knowledge of the results of the index tests?

\begin{tabular}{lll}
\hline & Low & Low \\
\hline
\end{tabular}

\section{DOMAIN 4: Flow and Timing}

Was there an appropriate interval Yes between index test and reference standard?

Did all patients receive the same Yes reference standard? 


\section{NCT01028053 (Continued)}

Were all patients included in the analysis?

Was the study with $18 \mathrm{~F}$ -

\section{Thurfjell 2012}

\section{Study characteristics}

Patient sampling

- Participants with $\mathrm{MCl}$ and Alzheimer's disease were included from 7 academic memory clinics and healthy volunteers were recruited by advertisement or they were the spouses of Alzheimer's disease patients or $\mathrm{MCl}$ participants.

- We only included data on the performance of the index test to discriminate between people with $\mathrm{MCl}$ who converted to dementia and those who remained stable.

- Among those participants, there were $20 \mathrm{MCl}$ participants. No further details of participant sampling and recruitment were reported.

- Inclusion criteria: MMSE 27 to 30 and older than 55 years (Vandenberghe 2010).

Patient characteristics and setting
- $20 \mathrm{MCl}$ participants diagnosed by the Petersen 1999 criteria. Demographic data were reported for $20 \mathrm{MCl}$ participants and they were classified as having amnestic $\mathrm{MCl}$.

- Gender: 11 male, 9 female.

- Mean \pm SD age: $72.7 \pm 7.09$ years (Vandenberghe 2010 )

- APOE $€ 4$ carrier: not reported

- MMSE: $28.0 \pm 0.94$ (Vandenberghe 2010)

- Mean \pm SD years of education: $14.8 \pm 2.97$ (Vandenberghe 2010)

- Sources of referral: not reported

- Setting: secondary care (memory clinic)
- PET imaging was conducted at 3 different scanning centres using a 16-slice Biograph PET/CT scanner (Siemens, Er- langen, Germany), an ECAT EXACT HR scanner (Siemens), and a GE Advance scanner, respectively.

- All PET time frames were realigned using an automated method and a PET sum image was created. The PET sum image was spatially normalised into Montreal Neurologic Institute space where a volume of interest (VOI) template was used to extract counts in VOIs for frontal, lateral temporal and parietal cortices as well as for the anterior and posterior cingulate. In addition, a reference region corresponding to the cerebellar cortex was defined.

- Standard uptake value ratios (SUVRs) were computed by dividing counts in the target regions with counts in the reference region. The authors computed a composite neocortical SUVR value as an average of the above mentioned cortical VOIs.

- 18 F-flutemetamol was injected intravenously as a slow bolus ( $<40$ seconds) in an antecubital vein (target activity set at $185 \mathrm{MBq}$ maximally (max), equivalent to an effective dose of approximately $6 \mathrm{mSv}$ ).

- 18F-flutemetamol administration mean MBq dose: 173.3 (SD 13.3).

- Time between ${ }^{18} \mathrm{~F}$-flutemetamol injection and PET acquisition: from 85 to 115 minutes $(6 \times 5$-minute frames).

- All PET time frames were realigned using an automated method and a PET sum image was created. The PET sum image was spatially normalized into Montreal Neurologic Institute space where a volume of interest (VOI) template was used to extract counts in VOIs for frontal, lateral temporal and parietal cortices as well as for the anterior and posterior cingulate. In addition, a reference region corresponding to the cerebellar cortex was defined. 
Thurfjell 2012 (Continued)

- Standard uptake value ratios (SUVRs) were computed by dividing counts in the target regions with counts in the reference region. The authors computed a composite neocortical SUVR value as an average of the above mentioned cortical VOls.

- Threshold: > 1.5 determined at baseline, based in Vandenberghe study with a threshold > 1.56 (Vandenberghe 2010).

- ROls included lateral frontal cortex (FRO), lateral temporal cortex (LTC), lateral parietal cortex (PAR), anterior cingulate (ANC), occipital cortex (OCC), and pons (PON).

- A cerebellar ROI served as reference region.

Target condition and reference standard(s)
- Target condition: Alzheimer's disease dementia.

- Reference standard: not explicitly stated, although NINCDS-ADRDA criteria for ADD (McKhann 1984) and APA 1994 were baseline diagnostic criteria.

Flow and timing

- Duration of follow-up: 2 years.

- Number included in analysis: 19 participants: 10 18F-flutemetamol (+) and 9 18F-flutemetamol (-)

- Progression from $\mathrm{MCl}$ to ADD:

- 10 18F-flutemetamol (+): $8 \mathrm{MCl}-\mathrm{ADD}$ and $2 \mathrm{MCl}-\mathrm{MCl} ; 9$ 18F-flutemetamol (-): $1 \mathrm{MCl}-\mathrm{ADD}$ and $8 \mathrm{MCl}-\mathrm{MCl}$

- $\mathrm{TP}=8 ; \mathrm{FP}=2 ; \mathrm{FN}=1 ; \mathrm{TN}=8$

- Loss to follow-up: $1 \mathrm{MCI}$ participant

- No further information was given

- Financial support for the baseline study (Vandenberghe 2010) was provided by the manufacturer of $18 \mathrm{~F}$ flutemetamol tracer and two authors of Thurfjell 2012 were employees

Comparative

Notes

\section{Methodological quality}

\begin{tabular}{llll}
\hline Item & Authors' judgement & Risk of bias & Applicability concerns
\end{tabular}

\section{DOMAIN 1: Patient Selection}

Was a consecutive or $\quad$ Unclear
random sample of pa-

tients enrolled?

\section{Was a case-control de- Yes}

sign avoided?

Did the study avoid in- Unclear

appropriate exclusions?

Unclear Low

\section{DOMAIN 2: Index Test All tests}

\section{Were the index test re- Yes} sults interpreted without knowledge of the results of the reference standard?

If a threshold was used, Yes was it pre-specified? 
Thurfjell 2012 (Continued)

Was the $18 \mathrm{~F}$-flutemetamol PET scan interpretation done by a trained reader physician?

Did the study provide a clear definition of what was considered to be a 18 F-flutemetamol positive result?

\begin{tabular}{lll}
\hline & Low & Low \\
\hline
\end{tabular}

\title{
DOMAIN 3: Reference Standard
}

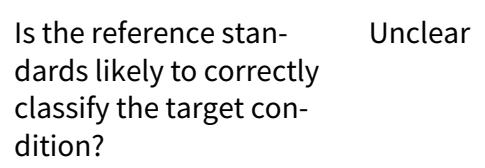

\begin{tabular}{lll}
\hline & Unclear & Unclear \\
\hline DOMAIN 4: Flow and Timing & &
\end{tabular}

\footnotetext{
Was there an appropri- Yes

ate interval between in-

dex test and reference

standard?
}

Did all patients receive Unclear
the same reference

standard?

\begin{abstract}
Were all patients in- Yes
cluded in the analysis?
\end{abstract}

Was the study with $18 \mathrm{~F}-\quad$ No

flutemetamol free of

commercial funding? 
FRO: Frontal cortex

LTC: Lateral temporal cortex

MBq: Megabecquerel

mcg: Microgramme

MCI: Mild cognitive impairment

MMSE: Mini-mental state examination

mSv: Millisievert

NINCDS-ADRDA: National Institute of Neurological and Communicative Disorders and Stroke and the Alzheimer's Disease and Related Disorders Association

OCC: Occipital cortex

PAR: Lateral parietal cortex

PET: Positron emission tomography

PON: Pons

ROI: Region of interest

SD: Standard deviation

SUVR: Standardised uptake value ratio

TN: True negative

TP: True positive

VOI: Volume of interest

Characteristics of excluded studies [ordered by study ID]

\section{Study \\ Reason for exclusion}

Goukasian 2015

Target condition: not looking at progression from MCI to dementia. The focus of the study was the association of $A \beta$ deposition and neuropsychiatric symptoms.

Rowe 2015a

Target condition: not looking at progression from MCl to dementia. The focus of the study was the change in ${ }^{18}$ F-flutemetamol PET scan retention over time.

Rowe 2015b

Target condition: not looking at progression from $\mathrm{MCl}$ to dementia. The focus of the study was the change in $18 \mathrm{~F}$-flutemetamol PET scan retention over time.

Rowe $2015 c$

Target condition: not looking at progression from MCl to dementia. The focus of the study was the change in $18 \mathrm{~F}$-flutemetamol retention over time.

$A \beta$ : Amyloid beta

MCl: Mild cognitive impairment

PET: Positron emission tomography

Characteristics of ongoing studies [ordered by study ID]

\section{EUCTR2011-001756-12-BE}

Trial name or title Surrogate markers evaluation in pre-demented Alzheimer's disease patients and healthy elderly controls

\section{Target condition and refer-} ence standard(s)

Progression to Alzheimer's disease at the end of the clinical follow-up period (from 1 to 3 years); no further details were given regarding the target condition(s) and the reference standard.

\begin{tabular}{ll}
\hline Index and comparator tests & 18 F-flutemetamol \\
\hline Starting date & April 2012 \\
\hline Contact information & Cliniques Universitaires Saint Luc, \\
& Nuclear Medicine Department,
\end{tabular}

18F PET with flutemetamol for the early diagnosis of Alzheimer's disease dementia and other dementias in people with mild cognitive impairment (MCl) (Review)

Copyright @ 2017 The Cochrane Collaboration. Published by John Wiley \& Sons, Ltd. 
EUCTR2011-001756-12-BE (Continued)

Dr R.Lhommel, renaud.lhommel@uclouvain.be

\section{Notes}

\section{EUCTR2011-006195-39-SE}

\section{Trial name or title}

An open-label study to compare the prognostic value of 18 F-flutemetamol PET scan imaging with longitudinal biomarker data in healthy volunteers and patients with mild cognitive impairment

\begin{tabular}{ll}
\hline $\begin{array}{l}\text { Target condition and refer- } \\
\text { ence standard(s) }\end{array}$ & $\begin{array}{l}\text { Progression to Alzheimer's disease and other dementias. No further details were given regarding } \\
\text { the target condition and the reference standard(s) used. Included subjects will be followed clinical- } \\
\text { ly over at least four years. }\end{array}$ \\
\hline Index and comparator tests & 18F-flutemetamol \\
\hline Starting date & February 2012 \\
\hline Contact information & Skånes universitetssjukhus, \\
& Minneskliniken, \\
& oskar.hansson@med.lu.se
\end{tabular}

Notes

\section{EUCTR2016-002635-15-NL}

\begin{tabular}{ll}
\hline Trial name or title & Study to Identify factors associated with resilience to clinical dementia at old age - 90+ study \\
\hline $\begin{array}{l}\text { Target condition and reference stan- } \\
\text { dard(s) }\end{array}$ & $\begin{array}{l}\text { Developing ADD in extremely elderly subjects; no further details were given regarding the } \\
\text { reference standard(s) used. }\end{array}$ \\
\hline Index and comparator tests & 18F-flutemetamol \\
\hline Starting date & July 2016 \\
\hline Contact information & Alzheimer Center, VU Medical Center \\
& n.legdeur@vumc.nl \\
\hline
\end{tabular}

Notes

\section{EUCTR2017-000094-36-SE}

Trial name or title

The BioFINDER 2 study - improved diagnostics and increased understanding of the pathophysiology of cognitive disorders

\section{Target condition and refer-} ence standard(s)

\section{Progression from subjective cognitive decline and $\mathrm{MCI}$ to $\mathrm{ADD}$ or other neurodegenerative disor-} ders; no further details were given regarding the reference standard(s) used.

Index and comparator tests $18 \mathrm{~F}$-flutemetamol

18F PET with flutemetamol for the early diagnosis of Alzheimer's disease dementia and other dementias in people with mild cognitive impairment (MCI) (Review)

Copyright @ 2017 The Cochrane Collaboration. Published by John Wiley \& Sons, Ltd. 
EUCTR2017-000094-36-SE (Continued)

\begin{tabular}{ll} 
Starting date & January 2017 \\
\hline Contact information & Minneskliniken, Skåne University Hospital \\
& Oskar.Hansson@med.lu.se
\end{tabular}

Notes

\section{JPRN-UMIN000019926}

\begin{tabular}{ll}
\hline Trial name or title & Clinical and neuroimaging study on preclinical Alzheimer's disease \\
\hline $\begin{array}{l}\text { Target condition and refer- } \\
\text { ence standard(s) }\end{array}$ & $\begin{array}{l}\text { Progression rate from asymptomatic preclinical ADD to } \mathrm{MCl} \text { and further to AD dementia at 36 } \\
\text { months of follow-up; no further details were given regarding the reference standard(s) used. }\end{array}$ \\
\hline Index and comparator tests & 11C-PiB, 18F-florbetapir or 18F-flutemetamol \\
\hline Starting date & January 2016 \\
\hline Contact information & Graduate School of medicine, Osaka City University, \\
& Center for Clinical study on dementia, \\
Hiroshi Mori, mori@med.osaka-cu.ac.jp
\end{tabular}

Notes

\begin{tabular}{ll}
\hline NCT02164643 & Longitudinal study of brain amyloid imaging in MEMENTO (MEMENTOAmyGing) \\
\hline $\begin{array}{l}\text { Target condition and refer- } \\
\text { ence standard(s) }\end{array}$ & $\begin{array}{l}\text { Progression to clinical dementia stage according to standardized classifications (DSM-IV and } \\
\text { NINCDS-ADRDA) at 2 years follow-up }\end{array}$ \\
\hline Index and comparator tests & 18F-flutemetamol and 18F-florbetapir at baseline \\
\hline Starting date & June 2014 \\
\hline Contact information & University Hospital, Bordeaux \\
& Prof. Geneviève Chene: genevieve.chene@isped.u-bordeaux2.fr \\
& Carole Dufouil: carole.dufouil@isped.u-bordeaux2.fr \\
\hline
\end{tabular}

\section{Notes}

\section{NCT02196116}




\section{NCT02196116 (Continued)}

Target condition and reference standard(s)

Index and comparator tests were prespecified.

\begin{tabular}{ll}
\hline Starting date & July 2014 \\
\hline Contact information & University Hospital, Bordeaux \\
& Michele Allard: michele.allard@chu-bordeaux.fr
\end{tabular}

Notes

ADD: Alzheimer's disease dementia

DSM-IV: Diagnostic and Statistical Manual of Mental Disorders (4th ed.)

$\mathrm{MCl}$ : Mild cognitive impairment

NINCDS-ADRDA: National Institute of Neurological and Communicative Disorders and Stroke and the Alzheimer's Disease and Related Disorders Association

\section{A T A}

Presented below are all the data for all of the tests entered into the review.

Table Tests. Data tables by test

\begin{tabular}{lll}
\hline Test & No. of studies & No. of participants \\
\hline 1 18F-flutemetamol & 2 & 243 \\
\hline
\end{tabular}

Test 1. 18F-flutemetamol.

\section{AP PE N DICES}

\section{Appendix 1. Glossary}

Aetiology: the cause, set of causes, or manner of causation of a disease or condition.

Amyloid beta $(\mathbf{A} \boldsymbol{\beta})$ : an amyloid that is derived from a larger precursor protein and is the primary component of plaques characteristic of Alzheimer's disease.

Biomarker: measurable and quantifiable biological parameters (e.g., specific enzyme concentration, specific hormone concentration, presence of biological substances) which serve as indices for health- and physiology-related assessments, such as disease risk, psychiatric disorders, environmental exposure and its effects, disease diagnosis; metabolic processes; etc.

Bolus: a single dose of a drug or other medicinal preparation given all at once.

Cingulate cortex: one of the convolutions on the medial surface of the cerebral hemispheres.

Cortical: the thin layer of grey matter on the surface of the cerebral hemispheres. It reaches its highest development in humans and is responsible for intellectual faculties and higher mental functions.

18F PET with flutemetamol for the early diagnosis of Alzheimer's disease dementia and other dementias in people with mild cognitive 34 impairment ( $\mathrm{MCI})$ (Review)

Copyright (c) 2017 The Cochrane Collaboration. Published by John Wiley \& Sons, Ltd. 
Epiphenomenon: A secondary effect or by-product. A secondary symptom or pathology, occurring simultaneously with a disease or condition but not directly related to it.

Frontotemporal: relating to the frontal and the temporal cerebral lobes.

Histopathology: the study of changes in tissues caused by disease.

Hypothyroidism: a syndrome that results from abnormally low secretion of thyroid hormones from the thyroid gland.

Index test: the test under evaluation.

In vivo: (of processes) performed or taking place in a living organism.

Ligand: a molecule that binds to another molecule, used especially to refer to a small molecule that binds specifically to a larger molecule, e.g., an antigen binding to an antibody, a hormone or neurotransmitter binding to a receptor, or a substrate or allosteric effector binding to an enzyme.

Neuritic plaques: accumulations of extracellularly deposited amyloid fibrils within tissues. Is one of the hallmarks of Alzheimer's disease.

Neurofibrillary tangles: abnormal structures located in various parts of the brain and composed of dense arrays of paired helical filaments (neurofilaments and microtubules). Are aggregates of hyperphosphorylated tau protein that are most commonly known as a primary marker of Alzheimer's disease.

Parietal lobe: upper central part of the cerebral hemisphere. It is located anterior to the occipital lobe, and superior to the temporal lobes.

Positron: an extremely small piece of matter with a positive electrical charge, having the same mass as an electron.

Precuneus: is a part of the parietal lobe of the brain, lying on the medial surface of the cerebral hemisphere.

Prodromal: Relating to prodrome; indicating an early stage of a disease.

Radionuclide (sometimes called a radioisotope or isotope): is a chemical which emits a type of radioactivity called gamma rays. The radioactivity can be detected by special scanners.

Reference standard: the best available method for establishing the presence or absence of the target condition.

Sensitivity: a measure of a test's ability to correctly detect people with the disease. It is the proportion of diseased cases that are correctly identified by the test. It is calculated as follows: Sensitivity = Number with disease who have a positive test/Number with disease.

Specificity: a measure of a test's ability to correctly identify people who do not have the disease. It is the proportion of people without the target disease who are correctly identified by the test. It is calculated as follows: Specificity = Number without disease who have a negative test/Number without disease.

Stilbene: organic compounds that contain 1,2-diphenylethylene as a functional group.

Target condition: the disease or condition that the index test is expected to detect.

Temporal lobe: lower lateral part of the cerebral hemisphere responsible for auditory, olfactory, and semantic processing. It is located inferior to the lateral fissure and anterior to the occipital lobe.

Vascular: relating to, affecting, or consisting of a vessel or vessels, especially those which carry blood.

\section{Appendix 2. Search strategy for 18 F-flutemetamol PET ligand}

\begin{tabular}{ll}
\hline Source & Search strategy \\
\hline MEDLINE In-process and oth- & 1. Flutemetamol.ti,ab,nm. \\
er non-indexed citations and & 2. (VIZAMYL or vizamyl*).ti,ab,nm. \\
MEDLINE ${ }^{\circledR} 1946$ to May 2017 (Ovid & SP) \\
& 3. "flutemetamol-fluorine-18".ti,ab,nm. \\
& 4. "18F-GE067".ti,ab,nm. \\
& 5. "[18F]Flutemetamol".ti,ab,nm. \\
\hline
\end{tabular}

$18 \mathrm{~F}$ PET with flutemetamol for the early diagnosis of Alzheimer's disease dementia and other dementias in people with mild cognitive 
(Continued)
6. "flutemetamol-PET".ti,ab,nm.
7. or/1-6
8. Fluorine Radioisotopes/du
9. Aniline Compounds/du
10. Ethylene Glycols/du
11. Stilbenes/du
12. Radioligand Assay/
13. radioligand ${ }^{*}$.ti,ab.
14. or/8-13
15. Alzheimer Disease/ri [Radionuclide Imaging]
16. Plaque, Amyloid/ri [Radionuclide Imaging]
17. or/15-16
18. 14 and 17
19. 7 or 18

Embase 1974 to May 2017 (Ovid $\mathrm{SP})$
1. Flutemetamol.ti,ab.

2. (VIZAMYL or vizamyl $\left.{ }^{\star}\right)$.ti,ab.

3. "flutemetamol-fluorine-18".ti,ab.

4. "18F-GE067".ti,ab.

5. "[18F]Flutemetamol".ti,ab.

6. "flutemetamol-PET".ti,ab.

7. exp flutemetamol $\mathrm{f} 18 /$

8. or/1-7

9. exp * radioligand/

10. Alzheimer disease/

11. Alzheimer ${ }^{\star}$.ti,ab.

12. amyloid plaque/di [Diagnosis]

13. mild cognitive impairment/

14. or/10-13

15. 9 and 14

16. 8 or 15
PsycINFO 1806 to May 2017 (Ovid
1. Flutemetamol.ti,ab.
$\mathrm{SP})$
2. (VIZAMYL or vizamyl $\left.{ }^{\star}\right)$.ti,ab.
3. "flutemetamol-fluorine-18".ti,ab.
4. "18F-GE067".ti,ab.
5. "[18F]Flutemetamol".ti,ab.
6. "flutemetamol-PET".ti,ab.
7. or/1-6

BIOSIS Citation Index (Thomson Reuters Web of Science) (1922 to May 2017)
Topic=(Flutemetamol OR VIZAMYL OR vizamyl ${ }^{\star}$ OR "flutemetamol-fluorine-18" OR "18F-GE067" OR "[18F]Flutemetamol" OR "flutemetamol-PET")

Timespan=All years. Databases $=\mathrm{BCl}$
Web of Science Core Collection, including the Science Citation
Topic=(Flutemetamol OR VIZAMYL OR vizamyl ${ }^{\star}$ OR "flutemetamol-fluorine-18" OR "18F-GE067" OR "[18F]Flutemetamol" OR "flutemetamol-PET")

18F PET with flutemetamol for the early diagnosis of Alzheimer's disease dementia and other dementias in people with mild cognitive 36 impairment ( $\mathrm{MCI})$ (Review)

Copyright (c) 2017 The Cochrane Collaboration. Published by John Wiley \& Sons, Ltd. 
(Continued)

Index and the Conference Proceedings Citation Index (Thomson Reuters Web of Science)

(1946 to May 2017)
Timespan=All years. Databases=SCI-EXPANDED, SSCl, A\&HCl, CPCI-S, CPCI-SSH, BKCl-S, BKClSSH, CCR-EXPANDED, IC.
Flutemetamol OR VIZAMYL OR vizamyl ${ }^{\star}$ OR "flutemetamol-fluorine-18" OR "18F-GE067" OR "[18F]Flutemetamol" OR "flutemetamol-PET" [Words]
CINAHL (EBSCOhost) (1980 to

May 2017)
S1 TX Flutemetamol

S2 TX VIZAMYL

S3 TX vizamyl ${ }^{*}$

S4 TX "flutemetamol-fluorine-18"

S5 TX "18F-GE067"

S6 TX "[18F]Flutemetamol"

S7 TX "flutemetamol-PET"

S8 S1 OR S2 OR S3 OR S4 OR S5 OR S6 OR S7

ClinicalTrials.gov (www.clinicaltrials.gov)

Flutemetamol OR VIZAMYL OR vizamyl ${ }^{\star}$ OR "flutemetamol-fluorine-18" OR "18F-GE067" OR "[18F]Flutemetamol" OR "flutemetamol-PET"

Flutemetamol OR VIZAMYL OR vizamyl OR "flutemetamol-fluorine-18" OR "18F-GE067" OR

"[18F]Flutemetamol" OR "flutemetamol-PET"
World Health Organization International Clinical Trials Registry Platform (WHO ICTRP) (http:// apps.who.int/trialsearch)

\section{ALOIS, the Cochrane Dementia \& \\ Imaging AND PET} Cognitive Improvement Group's specialized register of dementia studies (http://www.medicine.ox.ac.uk/alois/)

\section{Appendix 3. Tables $(2 \times 2)$ cross-relating index test results of the reference standards}

Table 1. Progression from mild cognitive impairment (MCI) to Alzheimer's disease dementia (ADD)

\begin{tabular}{|c|c|c|}
\hline \multirow[t]{2}{*}{ Index test information } & \multicolumn{2}{|l|}{ Reference standards information } \\
\hline & ADD present & ADD absent \\
\hline Index test-positive & $\begin{array}{l}\text { 18F-flutemetamol PET ligand for A } \beta \\
\text { (+) who progress to ADD (TP) }\end{array}$ & $\begin{array}{l}18 \text { F-flutemetamol PET ligand for } A \beta(+) \text { who remain } \mathrm{MCI}(\mathrm{FP}) \text { and } \\
18 \mathrm{~F} \text {-flutemetamol PET ligand for } \mathrm{A} \beta(+) \text { who progress to non-ADD } \\
\text { (FP) }\end{array}$ \\
\hline Index test-negative & $\begin{array}{l}\text { 18F-flutemetamol PET ligand for } A \beta \\
\text { (-) who progress to ADD (FN) }\end{array}$ & $\begin{array}{l}18 \text { F-flutemetamol PET ligand for } A \beta(-) \text { who remain } \mathrm{MCI}(\mathrm{TN}) \text { and } \\
18 \mathrm{~F} \text {-flutemetamol PET ligand for } \mathrm{A} \beta(-) \text { who progress to non-ADD } \\
\text { (TN) }\end{array}$ \\
\hline
\end{tabular}


ADD: Alzheimer's disease dementia

FN: False negative

FP: False positive

$\mathrm{MCl}$ : Mild cognitive impairment

PET: Positron emission tomography

TN: True negative

TP: True positive

Table 2. Progression from mild cognitive impairment $(\mathrm{MCI})$ to non-Alzheimer's disease dementia (non-ADD)

\begin{tabular}{|c|c|c|}
\hline \multirow[t]{2}{*}{ Index test information } & \multicolumn{2}{|l|}{ Reference standards information } \\
\hline & Non-ADD present & Non-ADD absent \\
\hline Index test-positive & $\begin{array}{l}\text { 18F-flutemetamol PET ligand for } A \beta \\
(+) \text { who progress to non-ADD (TP) }\end{array}$ & $\begin{array}{l}\text { 18F-flutemetamol PET ligand for } A \beta(+) \text { who remain } \mathrm{MCI}(\mathrm{FP}) \text { and } \\
\text { 18F-flutemetamol PET ligand } A \beta(+) \\
\text { who progress to ADD (FP) }\end{array}$ \\
\hline Index test-negative & $\begin{array}{l}\text { 18F-flutemetamol PET ligand for } A \beta \\
(-) \\
\text { who progress to non-ADD (FN) }\end{array}$ & $\begin{array}{l}\text { 18F-flutemetamol PET ligand for } A \beta(-) \text { who remain } \mathrm{MCI}(\mathrm{TN}) \text { and } \\
\text { 18F-flutemetamol PET ligand for } A \beta(-) \\
\text { who progress to ADD (TN) }\end{array}$ \\
\hline
\end{tabular}

ADD: Alzheimer's disease dementia

FN: False negative

FP: False positive

$\mathrm{MCl}$ : Mild cognitive impairment

PET: Positron emission tomography

TN: True negative

TP: True positive

Table 3. Progression from mild cognitive impairment $(\mathrm{MCl})$ to any form of dementia

\begin{tabular}{|c|c|c|}
\hline \multirow[t]{2}{*}{ Index test information } & \multicolumn{2}{|l|}{ References standard information } \\
\hline & Any forms of dementia present & Dementia absent \\
\hline Index test-positive & $\begin{array}{l}18 \mathrm{~F} \text {-flutemetamol PET ligand for } A \beta(+) \text { who progress to any } \\
\text { form of dementia (TP) }\end{array}$ & $\begin{array}{l}18 \mathrm{~F} \text {-flutemetamol PET ligand for } A \beta(+) \text { who } \\
\text { remain } \mathrm{MCl}(\mathrm{FP})\end{array}$ \\
\hline Index test-negative & $\begin{array}{l}18 \text { F-flutemetamol PET ligand for } A \beta(-) \text { who progress to any } \\
\text { form of dementia (FN) }\end{array}$ & $\begin{array}{l}\text { 18F-flutemetamol PET ligand for } A \beta(-) \text { who } \\
\text { remain } \mathrm{MCl}(\mathrm{TN})\end{array}$ \\
\hline
\end{tabular}


$\mathrm{MCl}$ : Mild cognitive impairment

PET: Positron emission tomography

TN: True negative

TP: True positive

Appendix 4. Assessment of methodological quality table: Quality Assessment of Diagnostic Accuracy Studies 2 (QUADAS-2) tool

\begin{tabular}{|c|c|c|c|c|}
\hline Domain & Patient selection & Index test & Reference standard & Flow and timing \\
\hline Description & $\begin{array}{l}\text { Describe methods of } \\
\text { patient selection: de- } \\
\text { scribe included patients } \\
\text { (prior testing, presenta- } \\
\text { tion, intended use of in- } \\
\text { dex test and setting) }\end{array}$ & $\begin{array}{l}\text { Describe the index } \\
\text { test } \\
\text { and how it was con- } \\
\text { ducted } \\
\text { and interpreted }\end{array}$ & $\begin{array}{l}\text { Describe the reference } \\
\text { standard and how it was } \\
\text { conducted and interpret- } \\
\text { ed }\end{array}$ & $\begin{array}{l}\text { Describe any patients who did } \\
\text { not receive the index test(s) or } \\
\text { reference standard, or both, or } \\
\text { who were excluded from } \\
\text { the } 2 \times 2 \text { table (refer to flow } \\
\text { diagram): describe the time } \\
\text { interval and any interventions } \\
\text { between index test(s) and refer- } \\
\text { ence standard }\end{array}$ \\
\hline
\end{tabular}

\begin{tabular}{|c|c|c|c|c|}
\hline \multirow[t]{4}{*}{$\begin{array}{l}\text { Signalling ques- } \\
\text { tions (yes/no/un- } \\
\text { clear) }\end{array}$} & $\begin{array}{l}\text { Was a consecutive or } \\
\text { random sample of pa- } \\
\text { tients enrolled? }\end{array}$ & $\begin{array}{l}\text { Were the index test } \\
\text { results interpreted } \\
\text { without knowledge } \\
\text { of the results of the } \\
\text { reference standard? }\end{array}$ & $\begin{array}{l}\text { Is the reference standard } \\
\text { likely to correctly classify } \\
\text { the target condition? }\end{array}$ & $\begin{array}{l}\text { Was there an appropriate inter- } \\
\text { val between index test(s) and } \\
\text { reference standard? }\end{array}$ \\
\hline & $\begin{array}{l}\text { Was a case-control de- } \\
\text { sign avoided? }\end{array}$ & \multirow{3}{*}{$\begin{array}{l}\text { If a threshold was } \\
\text { used, was it prespec- } \\
\text { ified? }\end{array}$} & \multirow{3}{*}{$\begin{array}{l}\text { Were the reference stan- } \\
\text { dard results interpreted } \\
\text { without knowledge of } \\
\text { the results of the index } \\
\text { test? }\end{array}$} & $\begin{array}{l}\text { Did all patients receive a refer- } \\
\text { ence standard? }\end{array}$ \\
\hline & \multirow[t]{2}{*}{$\begin{array}{l}\text { Did the study avoid in- } \\
\text { appropriate exclusions? }\end{array}$} & & & $\begin{array}{l}\text { Did all patients receive the } \\
\text { same reference standard? }\end{array}$ \\
\hline & & & & $\begin{array}{l}\text { Were all patients included in the } \\
\text { analysis? }\end{array}$ \\
\hline $\begin{array}{l}\text { Concerns regard- } \\
\text { ing } \\
\text { applicability } \\
\text { (high/low/un- } \\
\text { clear) }\end{array}$ & $\begin{array}{l}\text { Are there concerns that } \\
\text { the included patients } \\
\text { do not match the re- } \\
\text { view question? }\end{array}$ & $\begin{array}{l}\text { Are there concerns } \\
\text { that the index test, } \\
\text { its conduct, or inter- } \\
\text { pretation differ from } \\
\text { the review question? }\end{array}$ & $\begin{array}{l}\text { Are there concerns that } \\
\text { the target condition as } \\
\text { defined by the reference } \\
\text { standard does not match } \\
\text { the review question? }\end{array}$ & \\
\hline
\end{tabular}

Appendix 5. Anchoring statements for quality assessment of 18F-flutemetamol PET scan for A $\beta$ diagnostic studies

Table 4. Review question and inclusion criteria

\begin{tabular}{lll}
\hline Category & Review question & Inclusion criteria \\
\hline
\end{tabular}

18F PET with flutemetamol for the early diagnosis of Alzheimer's disease dementia and other dementias in people with mild cognitive impairment ( $\mathrm{MCI})$ (Review)

Copyright (c) 2017 The Cochrane Collaboration. Published by John Wiley \& Sons, Ltd. 
(Continued)

\begin{tabular}{|c|c|c|}
\hline Patients & $\begin{array}{l}\text { Participants with mild cognitive impair- } \\
\text { ment (MCI), no dementia }\end{array}$ & $\begin{array}{l}\text { Participants that fulfil the criteria for the clinical diagnosis of } \\
\mathrm{MCl} \text { at baseline }\end{array}$ \\
\hline
\end{tabular}

\begin{tabular}{ll}
\hline Index test & $\begin{array}{l}\text { 18F-flutemetamol PET ligand for A } \beta \text { bio- } \\
\text { marker }\end{array}$
\end{tabular}

\begin{tabular}{|c|c|c|}
\hline Target condition & $\begin{array}{l}\text { Alzheimer's disease dementia (ADD) (pro- } \\
\text { gression from } \mathrm{MCl} \text { to ADD) } \\
\text { Any other forms of dementia (progres- } \\
\text { sion from } \mathrm{MCl} \text { to any other forms of de- } \\
\text { mentia }\end{array}$ & $\begin{array}{l}\text { ADD (progression from } \mathrm{MCl} \text { to } \mathrm{ADD} \text { ) } \\
\text { Any other forms of dementia (progression from } \mathrm{MCl} \\
\text { to any other forms of dementia) }\end{array}$ \\
\hline Reference standard & $\begin{array}{l}\text { NINCDS-ADRDA; DSM; ICD; McKeith cri- } \\
\text { teria; Lund criteria; International Behav- } \\
\text { ioural Variant FTD Criteria Consortium; } \\
\text { NINDS-ARIEN criteria }\end{array}$ & $\begin{array}{l}\text { NINCDS-ADRDA; DSM; ICD; McKeith criteria; Lund criteria; } \\
\text { International Behavioural Variant FTD Criteria Consortium; } \\
\text { NINDS-ARIEN criteria }\end{array}$ \\
\hline Outcome & $\mathrm{N} / \mathrm{A}$ & Data to construct a $2 \times 2$ table \\
\hline Study design & $\mathrm{N} / \mathrm{A}$ & $\begin{array}{l}\text { Longitudinal cohort studies and nested case-control studies } \\
\text { if they incorporate a delayed verification design (case-con- } \\
\text { trol nested in cohort studies) }\end{array}$ \\
\hline
\end{tabular}

ADD: Alzheimer's disease dementia

DSM: Diagnostic and Statistical Manual of Mental Disorders

FTD: Frontotemporal dementia

ICD: International Classification of Diseases

$\mathrm{MCl}$ : Mild cognitive impairment

NINCDS-ADRDA: National Institute of Neurological and Communicative Disorders and Stroke and the Alzheimer's Disease and Related Disorders Association NINDS-AIREN: National Institute of Neurological Disorders and Stroke and Association Internationale pour la Recherché et l'Enseignement en Neurosciences PET: Positron emission tomography

\section{Anchoring statements for quality assessment ${ }^{18} \mathrm{~F}$-flutemetamol PET ligand for $A \beta$ diagnostic studies}

We have provided some core anchoring statements for quality assessment in the diagnostic test accuracy (DTA) review of the $18 \mathrm{~F}$ flutemetamol PET ligand for A $\beta$ biomarker in dementia. These statements are designed for use with the Quality Assessment of Diagnostic Accuracy Studies 2 (QUADAS-2) tool and are based on the guidance for quality assessment of DTA reviews of Informant Questionnaire on Cognitive Decline in the Elderly (IQCODE) in dementia (Quinn 2014). In assessing individual items, the score of unclear should only be given if there is genuine uncertainty. In these situations, we contacted the relevant study teams for additional information. Whenever we scored one question as high risk of bias, we considered the study as having a high risk of bias.

Table 5. Anchoring statements to assist with the 'Risk of bias' assessment

\begin{tabular}{lll}
\hline Question & $\begin{array}{l}\text { Response and weight- } \\
\text { ing }\end{array}$ & Explanation \\
\hline Patient selection & & \\
\hline $\begin{array}{l}\text { Was the sampling } \\
\text { method appropriate? }\end{array}$ & $\begin{array}{l}\text { No = high risk of bias } \\
\text { Yes = low risk of bias } \\
\text { Unclear = unclear risk } \\
\text { of bias }\end{array}$ & $\begin{array}{l}\text { Where sampling is used, the designs least likely to cause bias are consecutive } \\
\text { sampling or random sampling. Sampling that is based on volunteers or select- } \\
\text { ing subjects from a clinic or research resource is prone to bias. }\end{array}$ \\
\hline $\begin{array}{l}\text { Was a case-control or } \\
\text { similar design }\end{array}$ & $\begin{array}{l}\text { No = high risk of bias } \\
\text { Yes = low risk of bias }\end{array}$ & $\begin{array}{l}\text { Designs similar to case-control that may introduce bias are those designs } \\
\text { where the study team deliberately increase or decrease the proportion of sub- }\end{array}$ \\
\hline
\end{tabular}

18F PET with flutemetamol for the early diagnosis of Alzheimer's disease dementia and other dementias in people with mild cognitive 
(Continued)

avoided?

Unclear $=$ unclear risk of bias jects with the target condition, which may not be representative. Some casecontrol methods may already be excluded if they mix subjects from various settings.
Are exclusion criteria described and appropriate?
No $=$ high risk of bias Yes $=$ low risk of bias Unclear = unclear risk of bias
We automatically graded the study as unclear if the study authors did not detail exclusions (pending contact with study authors).

Where a study details exclusions, we graded the study as 'low risk' if we considered exclusions to be appropriate. Certain exclusions common to many studies of dementia are: medical instability; terminal disease; alcohol/substance misuse; concomitant psychiatric diagnosis; other neurodegenerative conditions.

Exclusions are not appropriate if they comprise 'difficult to diagnose' patients.

We labelled post-hoc and inappropriate exclusions as at 'high risk' of bias.

\section{Index test}

Was the 18 F-flutemetamol PET ligand for $A \beta$ biomarker's

assessment/interpretation performed without

knowledge of clinical dementia diagnosis?
No $=$ high risk of bias Yes $=$ low risk of bias Unclear = unclear risk of bias
Terms such as 'blinded' or 'independently and without knowledge of' are sufficient and full details of the blinding procedure are not required. Interpretation of the results of the index test may be influenced by knowledge of the results of the reference standard. If the index test is always interpreted prior to the reference standard, then the person interpreting the index test cannot be aware of the results of the reference standard and so this item could be rated as 'yes'.

For certain index tests, the result is objective and knowledge of the reference standard should not influence the result, e.g. level of protein in cerebrospinal fluid; in this instance, the quality assessment may be 'low risk' even if blinding was not achieved.
Was the 18 F-flutemetamol PET ligand for $A \beta$ biomarker's

threshold prespecified?
No $=$ high risk of bias Yes $=$ low risk of bias Unclear = unclear risk of bias
For scales and biomarkers, there is often a reference point (in units or categories) above which subjects are classified as 'test-positive'; this may be referred to as the threshold, clinical cut-off, or dichotomisation point. A study is classified at high risk of bias if the study authors define the optimal cut-off post-hoc based on their own study data because selecting the threshold to maximise sensitivity and/or specificity may lead to overoptimistic measures of test performance. Certain papers may use an alternative methodology for analysis that does not use thresholds and these papers should be classified as not applicable.
Was the 18 F-flutemetamol PET ligand for $A \beta$ scan interpretation done by a trained reader physician?
No $=$ high risk of bias Yes $=$ low risk of bias Unclear $=$ unclear risk of bias
If a trained reader physician performed the scan interpretation, we scored this item as 'yes'.

If no definition of trained reader was done, we scored this item as 'unclear'.

If a nontrained reader physician performed the scan interpretation, we scored this item as 'no'.
Did the study provide a clear definition of what was considered to be a 18 F-flutemetamol PET ligand for $A \beta$ biomarker's positive result?
No $=$ high risk of bias Yes $=$ low risk of bias Unclear $=$ unclear risk of bias

\section{If the study clearly stated the definition of a positive result (e.g. SUV), we scored this item as 'yes'.}

If the study did not give a definition of what it considered a positive result or the definition of a positive result varied between the participants, we scored this item as 'no'. item as 'unclear'.

\section{Reference standard}

18F PET with flutemetamol for the early diagnosis of Alzheimer's disease dementia and other dementias in people with mild cognitive impairment ( $\mathrm{MCI})$ (Review)

Copyright (c) 2017 The Cochrane Collaboration. Published by John Wiley \& Sons, Ltd. 
(Continued)

Is the assessment used for clinical diagnosis of dementia acceptable?
No $=$ high risk of bias Yes $=$ low risk of bias Unclear = unclear risk of bias
Commonly used international criteria to assist with clinical diagnosis of dementia included those detailed in DSM-IV and ICD-10.

Criteria specific to dementia subtypes included but were not limited to NINCDS-ADRDA criteria for Alzheimer's dementia; McKeith criteria for Lewy body dementia; Lund criteria and International Behavioural Variant FTD Criteria Consortium for frontotemporal dementia; and the NINDS-AIREN criteria for vascular dementia.

Where the criteria used for assessment were not familiar to the review authors or the Cochrane Dementia and Cognitive Improvement group ('unclear'), we classified this item as 'high risk of bias'.
Were clinical assessments for dementia performed without knowledge of the ${ }^{18} \mathrm{~F}$ flutemetamol PET ligand for $A \beta$ biomarker?
No $=$ high risk of bias Yes $=$ low risk of bias Unclear = unclear risk of bias
Terms such as 'blinded' or 'independently and without knowledge of' were sufficient and full details of the blinding procedure were not required. Interpretation of the results of the reference standard may be influenced by knowledge of the results of the index test.

\section{Patient flow}

Was there an appropriate interval between

18F-flutemetamol PET ligand for $A \beta$ biomarker and clinical dementia assessment?
No $=$ high risk of bias Yes $=$ low risk of bias Unclear = unclear risk of bias
As we test the accuracy of the $18 \mathrm{~F}$-flutemetamol PET ligand for $A \beta$ biomarker for $\mathrm{MCl}$ progression to dementia, there will always be a delay between the index test and the reference standard assessments. The time between the reference standard and the index test will influence the accuracy (Geslani 2005; Okello 2007; Visser 2006), and therefore we noted time as a separate variable (both within and between studies) and will test its influence on the diagnostic accuracy. We have set a minimum mean time to follow-up assessment of 1 year. If more than $16 \%$ of subjects have assessment for $\mathrm{MCI}$ progression before nine months, this item was scored 'no'.
Did all subjects get the same assessment for dementia regardless 18F-flutemetamol PET ligand for $A \beta$ biomarker?
No $=$ high risk of bias Yes $=$ low risk of bias Unclear = unclear risk of bias
There may be scenarios where participants who score 'test-positive' on the index test have a more detailed assessment. Where dementia assessment differs between participants, this should be classified as high risk of bias.

\section{Were all patients who received \\ 18F-flutemetamol PET ligand for $A \beta$ biomark- er's assessment includ- ed in the final analysis?}

No $=$ high risk of bias Yes $=$ low risk of bias Unclear $=$ unclear risk of bias
If the number of patients enrolled differs from the number of patients included in the $2 \times 2$ table, then there is the potential for bias. If patients lost to dropouts differ systematically from those who remain, then estimates of test performance may differ.

If there are dropouts, these should be accounted for; a maximum proportion of dropouts for a study to remain at low risk of bias has been specified as $20 \%$.
Were missing $18 \mathrm{~F}-$ flutemetamol PET ligand for $A \beta$ biomarker's results reported?
No $=$ high risk of bias Yes $=$ low risk of bias Unclear $=$ unclear risk of bias
Where missing or uninterpretable results are reported, and if there is substantial attrition (we have set an arbitrary value of $50 \%$ missing data), we will score this as 'no'. If the study did not report these results, we scored this as 'unclear' and we contacted the study authors.
Was the study with $18 \mathrm{~F}$ flutemetamol PET ligand for $A \beta$ biomarker free of commercial funding?
No $=$ high risk of bias Yes $=$ low risk of bias Unclear = unclear risk of bias
If the funding source is clearly stated and is not commercial, this should be scored as 'no'.

If the funding source is clearly stated and is commercial, this should be scored as 'yes'.

If not enough information is given to assess whether the funding source is commercial, the scored is 'unclear'.

18F PET with flutemetamol for the early diagnosis of Alzheimer's disease dementia and other dementias in people with mild cognitive 
(Continued)

\section{Anchoring statements to assist with assessment for applicability}

\begin{tabular}{|c|c|}
\hline Question & Explanation \\
\hline $\begin{array}{l}\text { Were included patients } \\
\text { representative of } \\
\text { the general population } \\
\text { of interest? }\end{array}$ & $\begin{array}{l}\text { The included patients should match the intended population as described in the review question. The re- } \\
\text { view authors should consider population in terms of symptoms; pretesting; potential disease prevalence; } \\
\text { setting. If there is a clear ground for suspecting an unrepresentative spectrum, the item should be rated } \\
\text { poor applicability. }\end{array}$ \\
\hline \multicolumn{2}{|l|}{ Index test } \\
\hline $\begin{array}{l}\text { Were sufficient data on } \\
18 \text { F-flutemetamol PET } \\
\text { ligand for } A \beta \text { biomark- } \\
\text { er's application given } \\
\text { for the test to be repeat- } \\
\text { ed in an independent } \\
\text { study? }\end{array}$ & $\begin{array}{l}\text { Variation in technology, test execution, and test interpretation may affect estimate of accuracy. In addi- } \\
\text { tion, the background, and training/expertise of the assessor should be reported and taken in considera- } \\
\text { tion. If } 18 \text { F-flutemetamol PET ligand for A } \beta \text { biomarker was not performed consistently, this item should be } \\
\text { rated poor applicability. }\end{array}$ \\
\hline \multicolumn{2}{|l|}{ Reference standard } \\
\hline $\begin{array}{l}\text { Was clinical diagnosis } \\
\text { of dementia made in a } \\
\text { manner similar to cur- } \\
\text { rent clinical practice? }\end{array}$ & $\begin{array}{l}\text { For many reviews, inclusion criteria and 'Risk of bias' assessments will already have assessed the demen- } \\
\text { tia diagnosis. For certain reviews, an applicability statement relating to the reference standard may not be } \\
\text { applicable. There is the possibility that a form of dementia assessment, although valid, may diagnose a far } \\
\text { larger proportion of people with disease than usual clinical practice. In this instance, the item should be } \\
\text { rated poor applicability. }\end{array}$ \\
\hline
\end{tabular}

DSM: Diagnostic and Statistical Manual of Mental Disorders

FTD: Frontotemporal dementia

ICD: International Classification of Diseases

$\mathrm{MCl}$ : Mild cognitive impairment

NINCDS-ADRDA: National Institute of Neurological and Communicative Disorders and Stroke and the Alzheimer's Disease and Related Disorders Association NINDS-AIREN: National Institute of Neurological Disorders and Stroke and Association Internationale pour la Recherché et l'Enseignement en Neurosciences PET: Positron emission tomography

\section{CONTRIBUTIONS OF AUTHORS}

- Gabriel Martínez, Robin WM Vernooij, and Paulina Fuentes Padilla: contributed to conception, design, and draft of the protocol; overall responsibility of study selection; data extraction; contacted the authors; draft of discussion; and authors' conclusion sections.

- Leon Flicker: contributed to conception, and designed and reviewed the draft protocol and final manuscript.

- Xavier Bonfill Cosp: reviewed the draft protocol and final manuscript.

- Javier Zamora: designed and drafted the protocol, performed statistical analyses, updated the statistical methods section and finalised the manuscript.

\section{DECLARATIONS OF INTEREST}

- Gabriel Martínez has no known conflicts of interest.

- Leon Flicker has no known conflicts of interest.

- Robin WM Vernooij has no known conflicts of interest.

- Paulina Fuentes Padilla has no known conflicts of interest.

- Javier Zamora has no known conflicts of interest.

- Xavier Bonfill Cosp has no known conflicts of interest. 


\section{SOURCES OF SUPPORT}

\section{Internal sources}

- No sources of support supplied

\section{External sources}

- National Institute for Health Research (NIHR), UK.

This protocol was supported by the NIHR, via Cochrane Infrastructure funding to the Cochrane Dementia and Cognitive Improvement group. The views and opinions expressed therein are those of the protocol authors and do not necessarily reflect those of the Systematic Reviews Programme, the NIHR, the NHS, or the Department of Health

\section{INDEX TERMS}

\section{Medical Subject Headings (MeSH)}

*Positron-Emission Tomography; Alzheimer Disease [*diagnostic imaging] [metabolism]; Amyloid; Aniline Compounds [economics] [ ${ }^{*}$ pharmacokinetics]; Benzothiazoles [economics] [ ${ }^{\star}$ pharmacokinetics]; Biomarkers; Cognitive Dysfunction [complications] [*diagnostic imaging] [metabolism]; Confidence Intervals; Disease Progression; Early Diagnosis; False Negative Reactions; False Positive Reactions; Follow-Up Studies; Radiopharmaceuticals [economics] [ ${ }^{*}$ pharmacokinetics]; Reference Standards; Sensitivity and Specificity; Time Factors

\section{MeSH check words}

Aged; Female; Humans; Male 